\title{
BUCHBESPRECHUNGEN
}

Allan, Danielle: Our Declaration

(Alois Riklin)....

Assmann, Dieter / Baasner, Frank / Wertheimer, Jürgen (Hg.), Ware Mensch - Die Ökonomisierung der Welt

(Georg Simet)....

Böhm, Enrico: Die Sicherheit des Westens

(Andreas Raffeiner)....

Hüstebeck, Momoyo: Dezentralisierung in Japan (Carmen Schmidt)........................ 223

Ionescu, Dana / Salzbborn, Samuel (Hg.):

Antisemitismus in deutschen Parteien

(Felix Sassmannshausen)...

Jesse, Eckhard / Liebold, Sebastian (Hg.):

Deutsche Politikwissenschaftler

(Bernd Mayerhofer).

Knelangen, Wilhelm / Stein, Tine (Hg.):

Kontinuität und Kontroverse

(Clemens Jesenitschnig).

Schweda, Mark: Entzweiung und Kompensation

(Martin Ingenfeld).

Sloterdijk, Peter: Die schrecklichen Kinder der

Neuzeit

(Karl-Heinz Nusser)

Weidenfeld, Werner: Europa. Eine Strategie

(Wolfgang Schlott).

Willke, Helmut: Regieren

(Helge F. Jani).
Danielle ALLAN: Our Declaration - A Reading of the Declaration of Independence in Defense of Equality; New York: Liveright Publishing, 2014, 315pp., $27.95 \$$

Danielle Allan, Afro-Amerikanerin, Professorin für politische Philosophie am Institute for Advanced Study in Princeton, hat ein wunderbares Buch geschrieben. So wie sie als Teenager im Kreis ihrer Familie zweimal die ganze Bibel Wort für Wort gelesen hat, so las sie nacheinander mit mehreren Studienjahrgängen, damals noch in Chicago, jeweils während eines ganzen Semesters, langsam, sorgfältig, samt Interpunktionen die 1337 Worte der amerikanischen Unabhängigkeitserklärung von 1776. Dabei verglich sie den ursprünglichen Entwurf von Thomas Jefferson mit dem zuerst von John Adams und Benjamin Franklin, dann vom Kontinentalkongress und schliesslich vom Typographen veränderten endgültigen Text. Der Kurs wurde doppelt geführt, tags für junge Undergraduates, nachts für lernbegierige Erwachsene mit und ohne Jobs. Vor allem in der Zusammenarbeit mit Letzteren erlebte sie eine persönliche Metamorphose und entdeckte in der Erklärung eine kohärente politische Philosophie.

So wie dem Rezensenten dürfte es den meisten Lesern des Buches wie Schuppen von den Augen fallen, die sich zuvor nicht die ganze Erklärung, sondern nur oder vor allem die gemeisselten Worte des Prologs eingeprägt haben: »We hold these truths to be self-evident, that all men are created equal, that they are endowed by their Creator with certain unalienable Rights, that among these are Life, Liberty and the pursuite of Happiness, - That to secure these rights, Governments are instituted among Men, deriving their just powers from the consent of the governed, - That whenever any Form of Government becomes destructive of these ends, it is the Right of the People to alter or to abolish it, and to institute new Government, laying its foundation of such principles and organizing its powers in such form, as to them shall seem most likely to effect their Safety and Happiness."

Allans Botschaft lautet: Equality ist in der Unabhängigkeitserklärung vom gleichen Rang wie Liberty; Gleichheit ist das Fundament der Freiheit. 
Doch die USA hätten im Lauf ihrer ganzen Geschichte die Gleichheit aller Menschen zu Gunsten eines Teils verraten. Das unerfüllte Gleichheitsversprechen verfolge die USA wie ein Schatten bis zum heutigen Tag. Allan kann es nicht ertragen, dass das Gleichheitsideal auf die Seite geschoben wurde und immer noch wird, bevor es seine volle Reife erlangt hat, und sie hofft, mit ihrer Defense of Equality nicht allein zu sein (23).

Allan entdeckt in der Unabhängigkeitserklärung fünf Fazetten des Gleichheitsprinzips, wobei dem Rezensenten vor allem drei einleuchten: Erstens die Gleichheit der individuellen Freiheitsrechte aller Menschen, zweitens die Gleichheit der politischen Teilnahmerechte aller Erwachsenen und drittens die Gleichheit der Souveränität der konföderierten Staaten im Verhältnis zu Großbritannien und allen Staaten der Erde (268f.).

Allan hält sich fast ausschließlich an die Quelle. Die Sekundärliteratur berücksichtigt sie kaum. Das ist überwiegend kein Nachteil. Dass sie es aber nicht für nötig hielt, wenigstens die Schriften von Jefferson und anderen Gründungsvätern zu Rate zu ziehen, war vielleicht doch keine so gute Idee. Hätte sie die Notes on the State of Virginia und die Briefe Jeffersons, berücksichtigt, dann hätte sie dessen Stellung zur Sklaverei nicht als »rätselhaft« bezeichnen können. Zwar erwähnt sie gebührend, dass Jeffersons Verurteilung der Sklaverei in der Urfassung der Unabhängigkeitserklärung vom Kontinentalkongress ersatzlos gestrichen worden ist. Auch aus anderen öffentlichen und privaten Äusserungen Jeffersons geht unmissverständlich hervor, dass er die Sklaverei als Unrecht verurteilt hat. Im Jefferson Memorial-Tempel am Potomac in Washington habe ich einst die Inschrift abgeschrieben: »Indeed I tremble for my country when I reflect that God is just; that his justice cannot sleep forever. «Das Zitat stammt aus den Notes on the State of Virginia. Aus dem Kontext geht klar hervor, dass Jefferson die Sklaverei im Sinn hatte. Er war grundsätzlich für die Abschaffung der Sklaverei. Aber wie? Weder die Rückschaffung nach Afrika noch die schlagartige Freilassung aller Sklaven war praktikabel, auch aus wirtschaftlichen Gründen, bestand doch die Bevölkerung in Jeffersons Heimatstaat zur Hälfte aus Sklaven. Nur eine sukzessive Freilassung kam in Frage. Deshalb ist es verständlich, dass Jefferson, abgesehen von den mit seiner Sklavin gezeugten Mischlingen, gegen Ende seines Lebens nur eine Handvoll seiner rund hundert Sklaven in die Freiheit entlassen hat. Gezittert hat er zu Recht, wenn man bedenkt, dass trotz der formellen Abschaffung der Sklaverei 1865 und der Bürgerrechtsgesetzgebung von 1965 die Diskriminierung der nichtweissen Bevölkerung in der Strafverfolgung, in der Justiz und im Alltag bis heute anhält.

Auch in Bezug auf das Los der Ureinwohner war Jefferson Realist. Er pflegte persönliche Kontakte mit Indianern in seiner Nachbarschaft, unternahm archäologische Ausgrabungen, studierte ihre Sitten und Gebräuche und behandelte sie mit Hochachtung. Eine Deportation in Gegenden westlich des Mississippi hielt er nicht für nachhaltig. Doch das Neben- und Miteinander verschiedener Kulturen und Sprachen konnte er sich nicht vorstellen. Blieb aus seiner Sicht nur die Assimilierung. Diesmal scheint es tatsächlich rätselhaft, warum Jefferson in der Unabhängigkeitserklärung die Urbevölkerung als »erbarmungslose Wilde brandmarkte, »deren Kriegführung durch unterschiedslose Vernichtung ohne Rücksicht auf Alter, Geschlecht und Umstände allgemein bekannt «sei, obwohl er sich in den Notes ganz anders äußerte. Warum haben John Adams und Benjamin Franklin, beide Mitglieder des mit der Ausarbeitung der Unabhängigkeitserklärung betrauten Fünfer-Komitees, dieses pauschale Verdammungsurteil im Entwurf Jeffersons durchgewinkt? Hat doch Adams in einem Brief an Jefferson über häufige Besuche eines Stammeshäuptlings in seinem Elternhaus berichtet. Und hatte doch Franklin die heute noch existierende Fünf-Stämme-Föderation in Kanada studiert, welche die bundesstaatliche Struktur der US-Verfassung von 1787 beeinflusst haben soll. Passte allen Beteiligten die bestensfalls nur halbe Wahrheit ins Konzept, um in der Sammelklage der Unabhängigkeitserklärung gegen Großbritannien den englischen König der Anstiftung bezichtigen zu können? Übrigens hinterlässt Allans Buch bezüglich des harten Schicksals der Urbevölkerung einen blinden Fleck.

Was den zweiten Aspekt des Gleichheitspostulats der Unabhängigkeitserklärung betrifft, das politische Teilnahmerecht aller Erwachsenen, verweigerten die USA das aktive und passive Wahlrecht während langer Zeit nicht nur den Sklaven und den Ureinwohnern, sondern auch den Frauen und den mittellosen weissen Männern. Im Gegensatz zu dieser Verletzung des Gleichheitsprinzips schrieb Jefferson um 1800 in einem Brief an 
J. Moor, er sei immer für das allgemeine Wahlrecht gewesen. Doch respektiere er die abweichende Meinung von Mitbürgern, welche glauben, ein gewisses Mass an Eigentum sei notwendig für die Unabhängigkeit des politischen Urteils. Freilich fügte er maliziös hinzu, er habe nie beobachtet, dass die Rechtschaffenheit der Menschen mit ihrem Reichtum wachse.

Auch der dritte Aspekt der Gleichheit, die gleiche Souveränität aller Staaten, wäre eine aktuellen Betrachtung wert. Dass die Großmacht USA nicht erst heute die Souveränität anderer Staaten, selbst unter Verletzung des Völkerrechts, durch die Übersteigerung des eigenen »nationalen Interesses « missachtet, ist für Allan kein Thema.

Gern hätte man von Allan mehr erfahren über den ideengeschichtlichen Hintergrund der Menschenrechte. Warum hat Jefferson in der Unabhängigkeitserklärung die ihm bestens bekannte Trilogie unveräußerlicher Menschenrechte »life, liberty and estate « von John Locke nicht übernommen, sondern das Eigentumsrecht (estate), zweifellos absichtlich, durch the pursuit of happiness ersetzt? Die Erklärung von Ray Forrest Harvey, Jefferson sei dazu vom Genfer Naturrechtsdenker Jean-Jacques Burlamaqui beeinflusst worden, hält einer quellenkritischen Prüfung kaum stand. Hatte der Großgrundbesitzer ein schlechtes Gewissen? Das Problem übermäßiger Ungleichheiten von Einkommen und Vermögen ist weder in der Unabhängigkeitserklärung noch im Buch von Allan thematisiert.

Ungeachtet der kritischen Anmerkungen, die Leistung von Danielle Allan ist ein überaus anregendes Musterbeispiel für wechselseitiges Lehren und Lernen von Dozenten und Studenten an einem geschichtsträchtigen und nach wie vor aktuellen Quellentext. Die Idee, ein Semester lang, gemeinsam mit den Studierenden, Wort für Wort, langsam und sorgfältig ein Manifest zu analysieren, das die Welt verändert hat und noch unausgeschöpftes Potential verspricht, ist inspirierend und motiviert zu eigenständiger Nachahmung.

Alois Riklin
Heinz Dieter ASSMANN, Frank BAASNER, Jürgen Wertheimer (Hg.): Ware Mensch - Die Ökonomisierung der Welt, Baden-Baden: Nomos, 2014, 241 S., 30,00 $€$

Der Band versammelt 14 Beiträge, die auf dem öffentlichen Forum des Projekts Wertewelten an der Universität Tübingen vom 17.-20.11.2010 gehalten wurden.

Leider fehlt eine Einführung der Herausgeber, in der nähere Angaben zum Projekt gemacht werden. Aus dem Video zu Ware Mensch. Die Ökonomisierung der Welt, das im Internet (auf wertewelten.net) zugänglich ist, geht jedoch hervor, dass das Thema aus der Perspektive Kulturen des Dialogs reflektiert werden sollte. Eingeladen wurden sowohl Literaturwissenschaftler als auch Juristen mit dem Ziel, eine Wertediskussion zu initiieren, die sowohl interkulturell die Regionen, aus der die eingeladenen Wissenschaftler kamen, als auch interdisziplinär deren Wertesysteme miteinander ins Gespräch zu bringen. Inwiefern dieser Austausch tatsächlich stattfand, ist aus dem Tagungsband jedoch nicht ersichtlich, da nur die (bereits vor über drei Jahren gehaltenen) Reden, teils im Original, teils modifiziert, abgedruckt sind. Zudem sind Texte, die sich mit der juristischen Sicht auf die Ökonomisierung befassen, stark unterrepräsentiert. Nur der fünfte und sechste Aufsatz thematisieren explizit den Zusammenhang von Recht und Literatur. Des Weiteren sind einige Aufsätze eher soziologisch denn literaturwissenschaftlich und/ oder juristisch orientiert und weisen kaum bzw. keinerlei Bezüge zu Quellen aus Literatur und/ oder Recht auf.

Im Eröffnungsbeitrag versucht Wertheimer aufzuzeigen, dass sich aus fiktionalen Texten die »Erstellung eines Täterprofils « von Protagonisten generieren lasse, die den Menschen auf eine rein ökonomische Größe reduzierten (23). Dieses Vorhaben zeigt bereits ein Hauptmanko des Buchs. Die Wertediskussion wird entweder stark personalisiert, idiographisch vorgetragen, indem sehr häufig einzelne Charaktere aus einzelnen, ausgewählten literarischen Werken untersucht werden.

Dominieren im Ansatz hingegen systematische Aspekte der Wertediskussion, entfernen sich die Autoren weitgehend vom literaturwissenschaftlichen und/oder juristischen Bezugsrahmen. So geht Kistner im zweiten Aufsatz des Buchs auf den $\mathrm{Zu}$ sammenhang »Freiheit und Demonstrativer Kon- 
sum «, insbesondere in Südafrika, ein. Die Bezugnahme auf den Roman Black Diamond von Zakes Mda zum Ende ihrer Ausführungen dient der Autorin lediglich zur Untermauerung ihrer »Klassenanalyse « (40). Warum ihr Beitrag nicht mit all den anderen Aufsätzen, die ebenfalls einen starken südafrikanischen Bezug aufweisen - aus regionaler Perspektive ist Südafrika gegenüber anderen Ländern/Ländergruppen/Staaten überrepräsentiert zusammen gruppiert wurde, ist nicht ersichtlich. Im Anschluss versucht Kimmich, »Guerilla-Taktiken gegen die Warenwelt « darzustellen. Die Beispiele, die sie aus Literatur und Film hierzu heranzieht, stammen aus der ersten Hälfte des letzten Jahrhunderts. Die anhand dieser Auseinandersetzung aufgezeigten Möglichkeiten, »einen echten Widerstand zu leisten ", sind denn "genuin ästhetische Angebote (55) und können aufgrund der vorgenommenen perspektivischen Einengung auch nur solche sein.

Auch Kreutzer wählt mit Brochs Schlafwandler ein Werk zum Gegenstand, das um »um 1930« entstand (59). Denn dieses Werk könne, seiner Ansicht nach, als Paradigma dafür dienen, "neuere Entwicklungen einer Ökonomisierung der Welt >kulturtheoretisch « zu erfassen. (59) Haupttriebfeder der damaligen wie heutigen, also jeder Wirtschaftswelt sei die von ihm unterstellte »Überbietungs-Logik [...] mit der Aussicht auf noch schnellere, noch höhere Gewinne.« (62)

Baur beschäftigt sich anschließend mit dem Bezug von »Law and Literature". Seiner Meinung nach liege der wesentliche Unterschied in der Verortung, nämlich darin, dass »Recht $[\ldots]$ topisch" (70), »Literatur hingegen [...] utopisch « sei, als "Literatur keinen festen Ort in unserem sozialen Leben « und damit »Freiheit in der Funktion« habe. (71) Zudem betrachtet Baur auch die »Verbindungslinie [...] von Ökonomie und Recht « und behauptet: »Die Ökonomie als solche ist wertfrei. Moralisches Empfinden ist in ihrem System, also ihrem Denken und Handeln nicht immanent. «(75) Verhaltensökonomische Studien widersprechen dem jedoch. Menschen handeln in bestimmten Situationen altruistischer, als das von Baur zu Recht als »eindimensional verkürzt « bezeichnete » homo oeconomicus «-Modell suggeriert. (76)

In Zusammenarbeit mit Lorenz versucht Baur danach, "Überzeugungs- und Gewissenstätern " in der "Spiegelung von juristischer Außensicht und literarischer Innenansicht ein Profil zu geben«.
(81) Die Autoren unterscheiden dabei zwei Typen: Dem einen gehe es »um vergeltende Wiederherstellung «, dem anderen »um die breite Bestätigung oder Implementierung eigener Werte.« (92f.) Einen Sonderfall letzteren Typs bildeten die Täter "pazifistischer Prägung, die ihre Fundamentalkritik [...] ausschließlich durch Eigenopfer beglaubigen «. (94) Doch so interessant die Ausführungen vom Ansatz her auch sind, einen dezidierten Bezug zum Thema Ware Mensch leisten sie nicht.

Takeda unterscheidet anschließend zwischen »Opferterrorismus « und »Terrorismusopfer « und zeigt exemplarisch auf, dass »der Mensch [in beiden Fällen] zum bloßen Mittel entwürdigt werden «kann. (104) Angesichts der »terroristische[n] Bedrohung « würden Menschenleben nach »einer notgedrungenen Kosten-Nutzen-Rechnung « taxiert: »Der Mensch bleibt Ware vor den fetischisierten Werten Demokratie, Freiheit und Sicherheit.«(106)

Unter Bezug auf Goethes Faust will Stassen sodann nachweisen, dass »Potenz, Beschleunigung (velocitas), und Liquidität « die »wichtigsten Drogen « des Protagonisten Faust sind. (115; im Original kursiv und fett gedruckt). Einzig die »Karriere Fausts als Ökonom [ende...] innerhalb des Dramas nicht mit [Fausts...] Versagen «. (121) Wie Goethes Faust sei auch »Balzacs César Birotteaus[“ ...] Krankheitsbild [...] der Anthropomorphisierung des Geldes geschuldet.« (136) Die Aufgabe der Literatur bestehe darin, "als Poesis die Theorie der Praxis « zu sein, (139) um - wie Marx formulierte - die »versteinerten Verhältnisse dadurch zum Tanzen [zu] zwingen, dass man ihnen ihre eigene Melodie vorsingt.« (140) Leider wird dieser Ansatz nicht weiter entfaltet.

Nagavajara sieht in Wagners Götterdämmerung den »Weg zur Verdinglichung der Menschheit [...] vorgezeichnet.« (146) In Büchners Woyzeck dann wandle »sich die Weltgeschichte in Richtung einer Ökonomisierung menschlichen Tuns«. (151) Insbesondere am »heutige[n] Starkult« zeige sich die Ökonomisierung als prozessimmanentes »Paradox «: "auf der einen Seite behandelt man die Stars wie Götter, aber auf der anderen Seite vermarktet man sie.« (160) Nicht nur »die Kunst «, sprich die Kunstwerke, sondern auch »die Künstler selbst [werden] kommodifiziert.« (162)

Amos hingegen versucht, statt konfrontativ wabre Bildung gegen Ware Bildung abzugrenzen, 
eine »Verständigung über widersprüchliche Tendenzen und Deutungsangebote « zu erzielen. (167) Auch sie weist auf die Ambivalenz innerhalb des Ökonomisierungsprozesses hin: Zwar sei »unbestreitbar, dass Bildung immer mehr in den Sog eines ökonomischen Kalküls gerät«, (168) doch »auch das Umgekehrte [gelte]: Ökonomisierungsprozesse schließen zweckfreie Aspekte von Bildung nicht aus.« (184)

Für Antoni ist die »Kommunikation zwischen den >Kulturen angesichts der heutigen Weltlage« gar von essentieller Bedeutung. (190) Hierzu sei "gegenseitiges Verstehen und Erklären« nötig, (190) das nur »über die Wahrnehmung der historischen Wandlungsfähigkeit und Dynamik « (199) von »Kultur als Wertesystem « (198) erfolgen könne.

Während Amos und Antoni keinen Bezug zu literarischen oder sonstigen künstlerischen Zeugnissen ausweisen, geht Adachi-Rabe zwar nicht auf literarische Zeugnisse, aber auf zwei Filme ein: Der Pianist von Roman Polanski und Gefabr und Begierde von Ang Lee sollen exemplarisch als »Repräsentationen des Historischen Materialismus « analysiert werden. Die eigentliche Intention dabei ist eine emanzipatorisch-aufklärerische: Die Filme bezweckten, dem Betrachter zu ermöglichen, seine eigene »materialistisch orientierte Denkweise zu erkennen und zu versuchen sich von dieser zu befreien «. (211)

Im vorletzten, relativ kurzen Beitrag versucht Deme, den Begriff "Neger zu dekonstruieren. Seiner Ansicht ist dieses Substantiv "ganz und gar nicht harmlos «. (218) Der Begriff und seine Denotate »bezeichnen $[\ldots]$ eine Ware, aber keine Menschen«. (218)

Zuletzt stellt sich von Maltzan die Frage, »wie und warum Werte, die in der neuen Verfassung Südafrikas festgelegt wurden, [...] entweder kaum Fuß fassen konnten oder bereits erodieren.« (226) Statt die Implementierung der neuen Werte voranzutreiben, mündete das Land bereits »in einen neuen Rassismus «, (227) nicht so sehr gegenüber Hell(-er-)bäutigen, sondern »insbesondere gegenüber Migranten aus anderen afrikanischen Ländern«. (229) Hierfür macht die Autorin ökonomische Schein-Gründe, nämlich »die (falsche) Wahrnehmung [verantwortlich], dass die Zugewanderten den Townshipbewohnern die Arbeitsplätze wegnähmen.« (236)

Fazit: Der Band enthält eine Menge interessanter Einzelaspekte, die aber kaum aufeinander be- zogen sind. Entgegen der ursprünglichen Intention des Projekts sind nicht in allen Beiträgen dezidiert literaturwissenschaftliche und/oder juristische Bezüge hergestellt und herausgearbeitet. Eine Auseinandersetzung mit (literarischen) Quellen, die explizit ökonomische Themen zum Gegenstand haben (z. B. Kaufmannsfamilien, Unternehmensführung und -alltag, Bankenkrise, Gewerkschaftsarbeit), erfolgt gar nicht. Doch nur so ließen sich wirtschaftsgeschichtliche, branchen- und u. a. auch managementkritische Meinungen in den Wertediskurs einbeziehen und mitbedenken. Sollte das Projekt fortgesetzt werden, sollte daher darauf geachtet werden, sowohl die Vielfältigkeit als auch die gegenseitige Durchdringung der Ansätze zu erhöhen.

Georg Simet

Enrico BÖHM: Die Sicherbeit des Westens. Entstehung und Funktion der G7-Gipfel (1975-1981) (=Studien zur Internationalen Geschichte; Bd.34); München: Oldenburg, 2014; ISBN, Münchent: Oldenbourg, 2014, 357 S., 49,95 $€$

Die Gipfeltreffen der sieben wichtigsten Industrienationen der Welt (Gruppe der Sieben, G7) trat erstmals 1975 zusammen und wurde 1998 mit der Aufnahme Russlands zur G8 erweitert. 2014 wurde Russlands Mitgliedschaft aufgrund der Krimkrise ausgesetzt, sodass man zum Format der G7 zurückkehrte. Deutschland spielt als eine hochentwickelte Industrienation bei den G7-Treffen und ihren Vereinbarungen eine tragende Rolle. Dies ist auf die Systematik der Außenpolitik und der Wirtschaftspolitik der Bundesrepublik zurückzuführen, die seit ihrer Gründung 1949 einen westorientierten Kurs verfolgt hat. Der BRD gelang es so, die durch die Besatzungsmächte eingeschränkten Handlungsmöglichkeiten schrittweise zu erweitern. Der Weltwirtschaftsgipfel der G7 ist nicht zuletzt das Resultat der Wirtschaftskrise der 1970er-Jahre, in der erkannt wurde, dass eine internationale Zusammenarbeit unausweichlich sei, um die Krise lösen zu können.

2008 veröffentlichte Johannes von Karczewski seine Studie »Weltwirtschaft ist unser Schicksal«: Helmut Schmidt und die Schaffung der Wirtschaftsgipfel, um die Frage zu beantworten, wie die Bonner Regierung die Wirtschaftskrise der 70erJahre des letzten Jahrhunderts gelöst hat. Den 
Schwerpunkt der erfolgreichen Krisenpolitik sieht der Autor in der verstärkten europäischen Zusammenarbeit und der Konsolidierung internationaler Beziehungen. Enrico Böhm schließt an diese Studie an und untersucht den Werdegang und die Funktion der G7-Gipfel von 1978 bis 1981. Dabei legt er Wert auf eine internationale Sichtweise. Der Autor bezieht sich in seiner Dissertation auf Quellen internationaler Archive, legt aber sein methodisches Vorgehen nicht offen, sodass dem Leser die Frage bleibt, wie intensiv Böhm mit diesen Quellen gearbeitet hat. Im Quellenverzeichnis findet der Leser den Hinweis auf die zwölf Archive, eine Auflistung der Befunde fehlt aber leider. Was auffällig ist, ist das Weglassen sämtlicher Akten des Auswärtigen Amts. Der Autor rechtfertigt dies mit der dreißigjährigen Schutzfrist, die ihm den Zugang zu den Quellen verwehren würde.

Böhm führt die weltweite wirtschaftliche Entwicklung seit 1945 detailliert aus und geht insbesondere auf den Zusammenbruch des Weltwährungssystems von Bretton Woods und die Erdölkrise ein. Die G7 sieht er als Ergebnis der Wirtschaftskrise und maßgeblich durch Helmut Schmidt initiiert, welcher die Wirkungen der Wirtschaftskrise der 1930er-Jahre bestens kannte. Eine politisch engere Kooperation auf internationaler Ebene hatte Schmidt bereits als Finanzminister unter Kanzler Willy Brandt angesteuert, weshalb auch der erste große Weltwirtschaftsgipfel 1975 stark von Schmidt geprägt wurde.

Das zweite Kapitel beinhaltet die Darstellung der Strukturen der G7. Der Autor berücksichtigt Mitgliederkonstellationen, skizziert die einzelnen Treffen und beschreibt die Arbeitskreise, welche in Form von Seminaren und »Kamingesprächen« stattgefunden haben. Nicht zu kurz kommt die Erörterung des Spannungsverhältnisses zwischen der G7 auf der einen und der Öffentlichkeit auf der anderen Seite. Im dritten Teil untersucht Böhm seine These, die Legitimationstechniken, mit denen die G7 versuchten, die Akzeptanz ihrer eigenen institutionellen Stellung im politisch-gesellschaftlichen System zu festigen. So konstatiert der Autor, dass Schmidt, Giscard und Ford die Wirtschaftspolitik als Bestandteil einer neuen globalen Sicherheitspolitik etabliert hätten, die auf gegenseitiger Abhängigkeit beruhe. Der Autor grenzt sich damit von den zeitgenössischen Stellungnahmen der Akteure ab, welche eine legitimationsstiftende Bedeutung der Gipfel stets bestritten. Böhm schlussfolgert darüber hinaus, dass die wirtschaftliche Entwicklung in den 1970er-Jahren als Schicksalsfrage gehandelt wurde, was für ihn ein vollkommen neues Phänomen der internationalen Kooperation darstellt. Doch dabei ist zu berücksichtigen, dass es schon im Vorfeld, so etwa 1948 bei Ludwig Erhard, Forderungen gegeben hat, die wirtschaftliche Entwicklung zu internationalisieren und auf Isolation und Protektionismus zu verzichten.

\section{Andreas Raffeiner}

Momoyo HÜSTEBECK: Dezentralisierung in Japan. Politische Autonomie und Partizipation; Wiesbaden: Springer, 2014, 227 S., 39,99 €

Beim vorliegenden Werk zur Dezentralisierungsreform in Japan, die von vielen als eine der spektakulärsten Reformen der japanischen Nachkriegszeit bezeichnet wurde, handelt es sich um eine Dissertation, die 2014 am Fachbereich Gesellschaftswissenschaften der Universität DuisburgEssen angenommen wurde. Mit der Verabschiedung Reform im Jahr 2000 wurde das System der zentralen Weisungsbefugnis an lokale Mandatsträger abgeschafft und durch ein neues System der Kooperation zwischen Zentralregierung und lokalen Verwaltungen ersetzt. Zudem wurden diverse direktdemokratische Elemente eingeführt, wie etwa lokale Referenden. Komplementiert wurde die Dezentralisierungsreform mit einer Gemeindestrukturreform, die zu einer Neuordnung der Gemeinden und damit zu einer deutlichen Verringerung ihrer Anzahl geführt hat. Schließlich wurde mit der Verabschiedung des NPO-Gesetzes ein neuer Rechtsrahmen geschaffen, der nunmehr Zulassung und Aktivitäten von NPOs verbindlich regelt. Diese Reformpakete waren in der Vergangenheit nicht nur in Japan, sondern auch im westlichen Ausland, Gegenstand zahlreicher Publikationen, wobei neben den eigentlichen Reformen und ihren konkreten Auswirkungen vor allem auf eine gestiegene lokale politische Partizipation der Bürger und auf die damit verbundenen Demokratisierungspotentiale verwiesen wurde.

Beinahe 20 Jahre nach den Reformen widmet sich Momoyo Hüstebeck dem Spannungsfeld zwischen effektiver Steuerung und der Stärkung lokaler politischer Partizipation (19). Hierbei wurden zwischen April und Juni 2008 in zwei Gemeinden 
des Großraums Tôkyô sowohl Vertreter der Zivilgesellschaft als auch Abgeordnete der Lokalparlamente und Experten befragt. Der Leser erfährt allerdings lediglich, dass »leitfadenorientierte Experteninterviews « (28) durchgeführt und die transkribierten Interviews in den Gemeinden mittels qualitativer Inhaltsanalyse im Hinblick auf Dezentralisierungsoutput, zivilgesellschaftlichem Einflusspotential etc. analysiert wurden (33f.). Hier hätte sich der Leser eine etwas eingehendere Betrachtung der Methodik und einen systematischen Output, etwa im Anhang, gewünscht. Möglicherweise wurde dies in der Dissertation genauer dargelegt; ob die Arbeit überarbeitet oder gekürzt wurde, bleibt allerdings offen. Wahl und Anzahl der Experten (225f.) sind aber als sehr gut zu bezeichnen.

Ausgewählt wurden die Stadt Mitaka, die als Vorbildkommune im Hinblick auf lokale Selbstverwaltung und Bürgerbeteiligung gilt und daher bereits Gegenstand zahlreicher Untersuchungen war, sowie Fujimi, eine »Schlafstadt «, die diesbezüglich im Vergleich als eher durchschnittlich gilt (31). Den theoretischen Rahmen der Arbeit bildet die komplexe Demokratietheorie von Fritz Scharpf, mittels derer das Spannungsfeld zwischen demokratischer Beteiligung und Effizienz der Reformen bewertet wurde (21).

Die Struktur und der Inhalt der Reformen sowie insbesondere die mangelnde eigene Finanzausstattung der japanischen Kommunen sind hinlänglich bekannt und würden keine weitere Publikation rechtfertigen. Innovativ ist das Buch aber, weil es der interessanten Frage nachgeht, ob mit einer Veränderung und Optimierung der Strukturen, wie etwa der Gemeindereform, und der Einführung direktpartizipatorischer Beteiligungsformen auch mehr Demokratie einhergeht, d.h. ob die Legitimationsdefizite, die im politischen System Japans augenfällig sind, damit behoben werden können (13f.). Seit Langem ist die politische Beteiligung in Japan rückläufig und das Maß an Politikverdrossenheit im internationalen Vergleich enorm hoch. In ähnlicher Weise wie in Deutschland ist insbesondere unter den Jüngeren eine stark nachlassende Partizipation erkennbar. Während sich bei nationalen Wahlen etwa $80 \%$ der über 60 Jährigen beteiligen, sind es unter den 20-jährigen weniger als die Hälfte. Auf der lokalen Ebene, auch das ist eine Parallele zu anderen entwickelten Industriestaaten, liegt die Beteilung nochmals deut- lich darunter. Kann also eine Stärkung der lokalen Beteiligungsmöglichkeiten diese Defizite ausgleichen?

Die deutliche Antwort ist: nein. Zwar betrachteten die beteiligten Befragten ihr Engagement als »ideellen Zugewinn", doch erachteten sie ihren tatsächlichen politischen Einfluss als gering (185). Vor allem die geringe Beteiligung der 20- bis 40jährigen ist auffällig (186). Da sich nur eine Minderheit beteilige, sei auch in Japan die Gefahr groß, dass sich Sonderinteressen bei politischen Entscheidungen durchsetzten (200). Die bei Scharpf bereits angelegte Kritik an einer unreflektierten Umsetzung von Dezentralisierung erwies sich damit als hilfreich, auch wenn die Autorin bemängelt, dass die Kritik am bedingungslosen Postulat der Mitbestimmung bei Scharpf mehr Raum einnehme, als die Entwicklung praktikabler Partizipationsansätze (200). Allerdings bewahrt dies die Studie vor der unreflektierten Begeisterung für die neuen Formen der Partizipation und der lokalen Demokratie in Japan, die früheren Studien eigen war.

Mehr lokale Partizipation ist somit kein Allheilmittel gegen Politikverdrossenheit, vor allem nicht unter den Jüngeren. Bezeichnend sind hierbei die Aussagen eines Aktivisten einer Bürgergruppe und eines Abgeordneten, die beide ihre Hoffung auf die gegenwärtig in Rente gehende, geburtenstarke Nachkriegsgeneration setzen (186). Auch wenn die Partizipation Älterer zu begrüßen ist, betonte doch schon Karl Mannheim, dass nur die Beteiligung der Jugend sozialen Fortschritt bedeute, da gegenwärtige Problematiken infolge des potentiell neuartigen Zuganges anders gedeutet und interpretiert werden und damit auch zu neuartigen Lösungskonzepten führen, während die ältere Generation bei ihrer früheren Orientierung verharre. Das als »Allheilmittel « gegen die Politikverdrossenheit gepriesene Mehr an lokaler Partizipation ist somit keinesfalls so wirksam, wie gedacht, vor allem nicht unter den Jüngeren. Politische Stagnation und Politikverdrossenheit haben sich dadurch nicht abgeschwächt.

Insbesondere in einer Schlafstadt wie Fujimi stellte sich zudem die Frage nach der lokalen Identifikation des postmodernen Individuums. So ergab die Studie, dass diese Pendler wenig Interesse zeigten, sich in ihrer Gemeinde politisch zu engagieren (186). Schon Ulrich Beck und Peter Sopp hinterfragten, ob es möglich sei, Sonntags Regio- 
nalismus und lokale Identitäten zu predigen und werktags eine flexible und mobile Gesellschaft einzuklagen.

Es ist ein großes Verdienst von Hüstebecks Studie, dass sie diese Problemlagen genau benennt und die Reformen nicht idealistisch, sondern realistisch analysiert. Auf der Agenda der vergleichenden Demokratieforschung steht daher die Frage nach einem zukunftsfähigen Konzept lokaler Mitbestimmung, das auch neue Formen der Partizipation einschließt und die sozio-ökonomischen Entwicklungen berücksichtigt.

Das Buch ist allen zu empfehlen, die sich in vergleichender Weise mit Dezentralisierung und Demokratie und dem Spannungsverhältnis zwischen institutionellen Rahmenbedingungen und bürgerlicher Partizipation befassen. Zwar gibt es keine Antwort auf die Frage, wie lokale politische Partizipation im 21. Jahrhundert aussehen soll, es benennt jedoch klar die Grenzen der Reform der derzeitigen Systeme lokaler Selbstverwaltung, insbesondere im Hinblick auf die Stärkung und Förderung der demokratischen Beteiligung der Bürger.

\section{Carmen Schmidt}

Dana IONESCU, Samuel SALZBORN (Hg.): Antisemitismus in deutschen Parteien, Baden-Baden: Nomos, 2014, 323 S., $59 €$

Vor allem der sekundäre Antisemitismus stellt in bundesdeutschen Parteien ein virulentes Problem dar. Dieses Fazit lässt sich aus dem Sammelband der Göttinger Politikwissenschaftler Dana Ionescu und Samuel Salzborn ziehen. Die Herausgeber richten den Blick auf einen bislang kaum bearbeiteten Themenkomplex. Dementsprechend formulieren sie in der Einleitung den Anspruch, gleichsam eine Leerstelle in der Parteien- und in der Antisemitismusforschung füllen zu wollen (9). Diese bestehe darin, dass das Verhältnis »der einzelnen Parteien " zum Antisemitismus bislang nicht in »systematisierender Weise u untersucht worden sei (9). Auch der Bericht zur empirischen Untersuchung des Antisemitismus in Deutschland Erscheinungsformen, Bedingungen, Präventionsansätze, der 2011 veröffentlicht wurde, berücksichtigt die deutschen Parteien insgesamt wenig mit Ausnahme der Linkspartei und der NPD, finden die anderen Parteien kaum Erwähnung. Da- rauf verweisen Julia Kopp und Tobias Neef in ihrem Beitrag (45). Dies ist Ausdruck des Problems, dass der Antisemitismus in Parteien, so Ionescu und Salzborn, meist nur im Hinblick auf "politische Randgruppen « untersucht werde (7). Doch spätestens in den 2000er Jahren habe sich in der Antisemitismusforschung die Erkenntnis durchgesetzt, dass Antisemitismus nicht nur ein »Phänomen der so genannten radikalen oder extremen Ränder « (8) darstellt. Hieran knüpfen die Herausgeber mit ihrem Sammelband an. In seinen insgesamt acht Beiträgen vermag der Band einen guten Eindruck von der Bedeutsamkeit der politikwissenschaftlichen Auseinandersetzung mit den Artikulationsformen des Antisemitismus in deutschen Parteien zu vermitteln.

Ausgewählt wurden die Parteien anhand der Frage, ob sie im Deutschen Bundestag oder zumindest in einem Landesparlament vertreten sind. Von den Herausgebern wurden demnach Fallstudien zur CDU (Julia Kopp und Tobias Neef), zur CSU (Bodo Kahmann), zur SPD (Sebastian Voigt), zum Bündnis 90/Die Grünen (Saskia Richter), zur Linkspartei (Martin Kloke), zur FDP (Teresa Nentwig und Franz Walter) zur Piratenpartei (Elke Wittich) und zur NPD (Patrick Gensing) zusammengetragen. Die Artikel machen einerseits deutlich, dass sich weit verbreitete antisemitische Ressentiments in den deutschen Parteien finden. Andererseits, so Ionescu und Salzborn, könnten die Parteien auch als Sprachrohr für antisemitische Äußerungen in der Gesellschaft dienen (300). Diese Äußerungen werden in allen Aufsätzen im Hinblick auf primäre und sekundäre Artikulationsformen des Antisemitismus differenziert.

Die primäre Form des Antisemitismus drücke sich, so der Antisemitismusforscher Bodo Kahmann in seinem Beitrag zur CSU, in Form von Stereotypen und Ressentiments aus, wie sie den modernen Antisemitismus seit dem neunzehnten Jahrhundert prägten. So wurden Juden mit der Zirkulationssphäre und dem Zinsgeschäft identifiziert (57). Diese Erscheinungsform stellte noch bis 1945 die dominante Artikulationsform dar. Im Postnazismus, so die beiden Parteienforscher Teresa Nentwig und Franz Walter in ihrem Aufsatz zur FDP, sei diese Form des Antisemitismus ins Private verdrängt und in der politischen Öffentlichkeit mit einer "kommunikativen Latenz « belegt worden (208). Die Autoren zeigen jedoch, wie zu Beginn der 2000er Jahre die FDP das Brechen 
von Normen und Regeln zu einem freidemokratischen Projekt machte, wodurch die »Phase privatisierter >Latenz< des Antisemitismus zu Ende ging « und manifeste Artikulationsformen des Antisemitismus Eingang in die öffentliche politische Debatte fanden (209). Die FDP-Kampagne zur Bundestagswahl 2002, die maßgeblich von Jürgen Möllemann geprägt wurde, habe zu diesem Wandel entscheidend beigetragen, so Nentwig und Walter weiter. Der primäre Antisemitismus trete in bundesrepublikanischen Parteien dennoch bis heute nur »im Einzelfall« auf (209). Einzige Ausnahme, so Patrick Gensing, sei die NPD, in der sich auch der manifeste Antisemitismus artikuliere (273).

Der sekundäre Antisemitismus trete hingegen, so Ionescu und Salzborn, nicht manifest, sondern in Form von Schuldabwehr, der Umkehr von Tätern und Opfern und der damit einhergehenden Entlastung auf (303). Sowohl die Schlussstrichforderungen der CDU (ebd.) als auch die von Bodo Kahmann konstatierte Täter-Opfer-Umkehr im Beitrag des CSU-Politikers Peter Gauweiler zur Goldhagen-Debatte zeugten von einem sekundären Antisemitismus (68). Formen dieses Antisemitismus ließen sich ebenfalls, so der Historiker Sebastian Voigt in seinem Beitrag zur SPD, in der Sozialdemokratie finden. Diesen macht Voigt an der im Jahr 2005 entbrannten »Heuschreckendebatte « fest. Der damalige SPD-Bundesvorsitzende Franz Müntefering hatte Finanzinvestoren wiederholt als »Heuschreckenschwärme« bezeichnet (106). Dennoch sei die SPD diejenige Partei im politischen System der Bundesrepublik Deutschland, in der sowohl historisch als auch aktuell ein differenzierter Umgang mit Antisemitismus vorherrsche. Historisch vorzuwerfen sei ihr vor allem, dass sie es vor und während des Nationalsozialismus nicht geschafft habe, »den Antisemitismus als eigenständiges Problem zu betrachten« (91).

Einen prominenten Ausdruck des sekundären Antisemitismus stellt die sogenannte Israelkritik dar. In diesem Zusammenhang verweist Martin Kloke auf die lange Tradition des israelbezogenen Antisemitismus in der Partei Die Linke, den er über die Vorgängerparteien PDS und SED in die DDR zurückverfolgt (154-155). Eindrücklich beschreibt Kloke den antisemitischen Gehalt der sogenannten Gaza-Flotille, an der auch die Linksparteiabgeordneten Annette Groth und Inge Höger teilgenommen haben. Diese waren an Bord der
Mavi Marmara, von deren Crew der Funkspruch »Go back to Auschwitz! « (163-164) gekommen sei. Der israelbezogene Antisemitismus, auch diesem Umstand kann der Sammelband Rechnung tragen, ist jedoch nicht nur in der Partei Die Linke virulent. Saskia Richter beschreibt in ihrem Aufsatz das Verhältnis von Bündnis 90/Die Grünen zum Antisemitismus. Dieses beleuchtet sie am Beispiel der Israelkritik, die vor allem in der Friedensbewegung, die die Parteibasis der Grünen maßgeblich geprägt habe, artikuliert werde. (123) So sei der Antrag auf Kennzeichnung israelischer Waren "aus den besetzten Gebieten « ein Hinweis auf den doppelten Maßstab, den die Partei in ihrer Beurteilung der Politik Israels anlege (134).

Der Ansatz, anhand von konkreten Fallstudien den Umgang der Parteien mit antisemitischen Äußerungen zu untersuchen, überzeugt an vielen Stellen und vermag auch zu zeigen, dass der Antisemitismus sich zwar immer in konkreten Einzelfällen ausdrückt, dass es sich dabei aber zugleich um ein strukturelles Problem handelt. So werde laut Elke Wittich, Redakteurin der Wochenzeitung Jungle World, der Antisemitismus in der Piratenpartei durch den Verweis darauf, dass es sich um Einzelfälle handle, relativiert (254 ff.). Dass die antisemitischen Vorfälle keine Konsequenzen nach sich zögen, verweise auf das strukturelle Problem der Partei, Antisemitismus weder hinreichend erkennen noch adäquat kritisieren zu können (266).

Der Antisemitismus, so ließe sich mit Ionescu und Salzborn resümieren, stellt für die deutschen politischen Parteien demnach immer nur dann ein Problem dar, wenn es die anderen Parteien betrifft (314). Daher reiche die Feststellung anti-antisemitischer Positionierungen der Parteien nicht aus (315) - sie müssten vielmehr auf ihren qualitativen Gehalt hin untersucht werden. Konsequenzen aus antisemitischen Äußerungen würden selten und auch nur dann gezogen, wenn ein entsprechender öffentlicher Druck aufgebaut werde (314). Die entscheidende Ausnahme stellt dabei die NPD dar, die den Antisemitismus nicht als Problem, sondern als Teil ihrer Weltanschauung auffasst.

Mit diesem Resümee gelangt man jedoch an eine entscheidende Schwachstelle des Sammelbandes, die zwangsläufig auch seiner Form geschuldet ist. Der Maßstab dafür, ab wann latenter Antisemitismus in deutschen Parteien in manifesten Antisemitismus umschlägt, scheint bei den Autoren un- 
terschiedlich zu sein. Insofern ist zu konstatieren, dass den Aufsätzen ein gemeinsamer Antisemitismusbegriff fehlt, wodurch der Band an Kohärenz einbüßt. Dies lässt sich insbesondere am israelbezogenen Antisemitismus nachvollziehen: So begreift Martin Kloke in seinem Beitrag zur Linkspartei das Verhältnis von Antizionismus und Antisemitismus als »dialektisches Verhältnis« (179) Saskia Richter hingegen führt in ihrem Artikel zur Partei Bündnis 90/Die Grünen keine grundlegende Bestimmung zu diesem Verhältnis ein. Dieser Umstand mündet in den Beiträgen mal in einen breit gefassten und mal in einen eng gefassten Antisemitismusbegriff.

Ein gemeinsamer Maßstab für die Beurteilung des Antisemitismus in deutschen Parteien wäre für die weitere wissenschaftliche Auseinandersetzung jedoch richtungsweisend. Insofern kann der Band dem eigenen Anspruch, den Antisemitismus in deutschen Parteien systematisierend zu erforschen, nicht in Gänze gerecht werden. Die analytische Heterogenität der Aufsätze verweist somit auf die Leerstelle, die der Band zu füllen beansprucht. Trotz dieser Schwäche bietet der Sammelband einen differenzierten und eindrücklichen Beitrag zur Antisemitismus- und Parteienforschung. Insbesondere der Aufsatz von Sebastian Voigt zur SPD zeugt - aufgrund seiner Verknüpfung von historischer und theoretischer Analysevom Potenzial einer interdisziplinären systematischen Analyse des Antisemitismus in deutschen Parteien. Insofern lässt der Band auf eine weiterführende wissenschaftliche Debatte und Veröffentlichungen zu dem Thema hoffen, vor allem in Anbetracht des aktuell auflodernden israelbezogenen Antisemitismus im Rahmen der Pro-Gaza Demonstrationen.

\section{Felix Sassmannshausen}

Eckhard JESSE / Sebastian LIEBOLD (Hg): Deutsche Politikwissenschaftler - Werk und Wirkung; Baden-Baden: Nomos, 2014, 849 S., 98,00 €

Aus der Vorrede der Hegelschen Rechtsphilosophie stammt der bekannte Satz: »Wenn die Philosophie ihr Grau in Grau malt, dann ist eine Gestalt des Lebens alt geworden, und mit Grau in Grau lässt sie sich nicht verjüngen, sondern nur erkennen; die Eule der Minerva beginnt erst mit der einbrechenden Dämmerung ihren Flug. « Gemeint hat
Hegel damit, dass wahre Erkenntnis, sprich: die Erkenntnis dessen, was ist, erst aus einem gewissen zeitlichen Abstand heraus möglich sei. Erst wenn die zu erklärenden Phänomene selber bereits Geschichte geworden seien, könne die >dämmerungsaktive< Philosophie sie mit begründeter Aussicht auf Erfolg zu begreifen versuchen. Man mag darüber streiten, ob die Philosophie damit zutreffend charakterisiert ist. Nicht bestreiten lässt sich, dass jeder ernsthafte historische Erklärungsversuch den zeitlichen Abstand zu den Phänomenen voraussetzt. Hier gilt in der Tat Hegels Feststellung, dass eine Gestalt des Lebens alt geworden sein muss, damit die Geschichte »ihr Grau in Grau« malen kann!

Es scheint so, als sei für die bundesdeutsche Politikwissenschaft dieser Zustand, seit längerem schon, erreicht. Das seit Mitte der 1980er Jahre kontinuierlich gewachsene Interesse deutscher Politikwissenschaftler an der Vergangenheit ihrer Disziplin - seinen deutlichsten Niederschlag hat es in Wilhelm Bleeks monumentaler Studie Geschichte der Politikwissenschaft in Deutschland (2001) gefunden - deutet jedenfalls in diese Richtung. Nun liegt mit dem anzuzeigenden Sammelband eine neue, nicht minder monumentale Publikation vor, die als ein weiterer wissenschaftsgeschichtlicher Mosaikstein gelten darf. Wie der Titel verrät, haben es die Herausgeber diesmal nicht auf eine historische Gesamtdarstellung der Fachgeschichte, sondern auf deren subjektiv-biographische Dimension abgesehen. Es ist fürwahr ein Gemeinplatz, dass Geschichte von Menschen und Wissenschaft von Wissenschaftlern gemacht wird (freilich nicht ausschließlich) - weshalb eine die Akteursperspektive in den Vordergrund rückende Darstellung auch keiner längeren Begründung bedarf. Begründungspflichtig ist in diesem Fall allein die Auswahl der Porträtierten, und auf deren Erläuterung verwenden die an der TU Chemnitz lehrenden Herausgeber in ihrer sehr umfangreich geratenen, knapp 70 Seiten starken Einleitung, die selbst eine kleine Fachgeschichte darstellt, denn auch und verständlicherweise viel Zeit und Raum.

Machen wir es kurz: Sowohl der Kriterienkatalog (fachliche Kompetenz, erfolgreiches Wissenschaftsmanagement inklusive Bildung einer Schule, öffentliche Sichtbarkeit sowie - fakultativ - ein Mindestalter von 70 Jahren) als auch die getroffene Personenauswahl überzeugen. Dass freilich »bei einer Anzahl von 50 Personen ... Dissens präjudi- 
ziert (ist)«(18), räumen die Herausgeber selber ein. Jede Auswahl stellt immer auch eine Entscheidung dar, und es ist das gute Recht der Verantwortlichen, diese Entscheidung nach eigenem Ermessen $\mathrm{zu}$ treffen. In jedem Fall ist es Jesse und Liebold zugute zu halten, dass sie sich um ein Höchstmaß an Transparenz und »intersubjektive(r) Nachvollziehbarkeit« (30) bemüht haben: Sie diskutieren ihre Entscheidungen, zeigen Dilemmas und Härtefälle auf, begründen Ausnahmen und Ablehnungen - und nehmen damit einer möglichen Kritik den Wind aus den Segeln. Dass sich unter den Porträtierten mit Helga Haftendorn, Beate KohlerKoch und Gerda Zellentin nur drei Frauen befinden, ist am wenigsten den Herausgebern anzulasten, sondern Ausdruck der bis in die Gegenwart fortwirkenden Männerdomäne des Faches!

Was für die Auswahl der Porträtierten gilt, trifft auch auf die kaum weniger schwierige Auswahl der Autoren zu, zu denen pikanterweise Politikwissenschaftler gehören, die selber eine Würdigung erfahren. $\mathrm{Zu}$ nennen wären hier Klaus von Beyme, Jürgen W. Falter, Joachim Jens Hesse, Max Kaase, Peter Graf Kielmansegg, Beate KohlerKoch, Herfried Münkler, Franz Urban Pappi und Manfred G. Schmidt. Es ist das Verdienst der Herausgeber, dass weder diese Personalunion noch die unvermeidlichen »Nahverhältnisse «(31) zwischen Porträtierten und Porträtierenden dem Projekt ernsthaft geschadet haben. Mit Ausnahme eines einzigen Falles, auf den Jesse/Liebold in der Einleitung, wenngleich ohne Nennung von Namen, selbst hinweisen, versammelt der Band gleichermaßen informative, um wissenschaftliche Objektivität wie faire Bewertung bemühte, durchweg gut lesbare Texte, die sich in der Summe zu einem repräsentativen, keineswegs hegel-grauen, sondern ungemein bunten Bild der deutschen Politikwissenschaft seit 1945 verdichten. Dass die Beiträge über Lebende fast ausnahmslos wohlwollender ausgefallen sind als die über verstorbene Personen, liegt in der Natur der Sache. Ein "panegyrischer Ton « wird - dank des beherzten redaktionellen $\mathrm{Zu}$ - und Eingriffs - jedoch kaum irgendwo angeschlagen.

Die Beiträge selbst zeichnen sich, der besseren Vergleichbarkeit wegen, durch eine einheitliche Gliederung aus: Auf einen kurzen Vorspann folgen drei Kapitel zur Vita, zu den (thematisch im Vordergrund stehenden) Forschungsschwerpunkten sowie zu Schulgründung und Wissenschafts- management. Eine »kritische Würdigung« sowie ein Abschnitt über die Rezeption im Fach und in der Öffentlichkeit beschließen jeden Beitrag. Die beigefügte Auswahlbibliographie, die sowohl Primär- als auch Sekundärliteratur aufführt, eröffnet interessierten Nutzern erste Zugänge zur Vertiefung des Gelesenen (Gleiches gilt, nebenbei bemerkt, auch für das von den Herausgebern zusammengestellte Verzeichnis der Festschriften und die ebenfalls ans Ende der Einleitung angehängte Literaturübersicht $\mathrm{zu}$ deutschen Politikwissenschaftlern). Auch wenn man selbst im einen oder anderen Fall die Akzente gern ein wenig anders gesetzt gesehen hätte, so taugt diese Feststellung doch nicht als Kritik, da das Setzen von Akzenten zu den Rechten eines jeden Autors gehört und im Übrigen, aller intendierten Objektivität und Fairness zum Trotz, unvermeidlich ist!

Wer sich nicht nur in einzelne Beiträge vertieft, sondern den gesamten Band durcharbeitet - was freilich mit einem erheblichen Zeitaufwand verbunden wäre und daher eher die Ausnahme als die Regel sein dürfte -, wird auf's Neue eindrucksvoll bestätigt finden, was den Reichtum der deutschen Nachkriegs-Politikwissenschaft ausmacht: die Vielfalt an Themen, Perspektiven, Fragestellungen sowie theoretischen und methodischen Ansätzen. Und sich des Eindrucks nicht erwehren können, dass eben diese Vielfalt in der Folge der Durchsetzung der Politikwissenschaft als akademischem Lehrfach und ihrer Transformation in eine »normal science « nach amerikanischem Vorbild, allen neu besiedelten Lehr- und Forschungskontinenten (Entwicklungsforschung, Friedens- und Konfliktforschung, empirische Wahlforschung etc.) zum Trotz, verloren gegangen ist. Die »Versozialwissenschaftlichung «- die ja gleichbedeutend mit der Herauslösung der Politikwissenschaft aus dem Rahmen der Geisteswissenschaften war - hat fraglos das empirische Theorie- und Methodenprofil der Disziplin geschärft, jedoch auf Kosten der Verbindung zu den Traditionen der älteren Politiklehre vor 1900, die für die Gründergeneration noch selbstverständlich war, sowie auf Kosten ihrer gesellschaftlichen Bedeutsamkeit. Wie bereits Bleek es tat, so warnen daher auch die Herausgeber am Ende ihrer Einleitung vor einer überzogenen empirisch-sozialwissenschaftlichen Profilierung: "Das Fach ist gut beraten, sich weniger um Szientismus als um Verständlichkeit zu bemühen, weniger die Methoden als Selbstzweck zu betreiben, 
in der Lehre auf Aneignung von Wissen, öffentlich auf mehr politische Bildung und im Fach wie in der schulischen Bildung stärker auf Urteilskraft zu setzen.«(69)

Fazit: Das hier besprochene Kompendium ist ein »Band von Politikwissenschaftlern über Politikwissenschaftler « (9), der einen wichtigen und, allein aufgrund des schieren Umfangs, 'gewichtigen Beitrag sowohl zur Geschichte als auch zur Gegenwart des Faches liefert. Ein Band, der die Gründungsväter der deutschen Politikwissenschaft (u.a. Wolfgang Abendroth, Arnold Bergstraesser, Theodor Eschenburg, Ernst Fraenkel, Carl Joachim Friedrich, Ossip K. Flechtheim, Ferdinand A. Hermens, Siegfried Landshut, Richard Loewenthal und Dolf Sternberger) angemessen repräsentiert, darüber jedoch auch die nachfolgende zweite und dritte Wissenschaftsgenerationen nicht vergisst. Ein Band nicht zuletzt, der - vermittelt über die Personen - neben den großen Teilgebieten der Disziplin (Innenpolitik, Vergleichende Regierungslehre, Politische Theorie, Internationale Politik etc.) auch die neueren Forschungsansätze (Policy-, Governance-, EU- und Transformationsforschung) abbildet, lexikalischen Anspruch und essayistische Form zwanglos miteinander verbindet, als biographisches Handwörterbuch ebenso wie als politikwissenschaftliches bzw. fachhistorisches Nachschlagewerk genutzt werden kann und daher in keiner politikwissenschaftlichen Bibliothek fehlen sollte.

\section{Bernd Mayerhofer}

Wilhelm KNELANGEN / Tine STEIN (Hg.): Kontinuität und Kontroverse. Die Geschichte der Politikwissenschaft an der Universität Kiel; Essen: Klartext Verlag, 2013, 522 S., 29,95 €

Der Sammelband Kontinuität und Kontroverse vereint Forschungserträge anlässlich des 60-jährigen Jubiläums der akademischen Disziplin Politikwissenschaft an der Christian-Albrechts-Universität zu Kiel (CAU). Chronologisch gegliedert wird in 19 Beiträgen ein »historisch großer Bogen gespannt « (13), der von der älteren Lehre der Politik im 17. und 18. Jahrhundert bis in die jüngste Vergangenheit reicht.

In ihrer Einleitung lassen Stein und Knelangen die Geschichte der Politikwissenschaft in Deutschland und an der CAU Revue passieren und stellen die These auf, dass sich "anhand der Universität im Norden nahezu exemplarisch die Entwicklungsgeschichte der Politikwissenschaft in Deutschland aufzeigen [lässt] « (12). Dies soll im Weiteren »vornehmlich entlang der Geschichte der Personen « (13) geschehen, die an der CAU tätig waren (leider fehlt ein entsprechendes Register).

Die erste historische Abteilung behandelt in sechs Beiträgen die Anfänge einer Lehre von der Politik an der CAU vom 17. bis ins 19. Jahrhundert. Wilhelm Knelangen und Marco Lück skizzieren am Beispiel dreier Professoren, wie sich das Politikverständnis wandelte. Im 18. Jahrhundert befand sich die CAU zeitweilig im Niedergang, weshalb die Staatswissenschaften zunächst nur mit einer einzigen ordentlichen Professur bedacht waren, auf der über 40 Jahre August Christian Heinrich Niemann prägend wirkte, wie Knelangen zeigt. Der Staatsrechtler Niels Nicolaus Falck wirkte kaum minder lang in Kiel; er weise mit seinem modernen Verfassungsdenken über seine Zeit hinaus, so Utz Schliesky in seinem Beitrag. Wilhelm Bleek stellt einen Zeitgenossen Falcks vor, der wie dieser vehement die Position von der Unteilbarkeit Schleswig-Holsteins vertrat: Friedrich Christoph Dahlmann. Für ihn war ebenso wie für Johann Gustav Droysen selbstverständlich, dass Geschichtswissenschaft und Lehre von der Politik eng miteinander verwoben seien. In einem Beitrag über Lorenz von Stein argumentiert Joachim Krause, dass dieser Denker uns auch heute noch viel zu sagen hat: als Politiktheoretiker wie als politischer Philosoph, der ein »neues Verständnis vom Sozialvertrag « (208) angedacht habe.

Die zweite historische Abteilung - Kaiserreich und Republik - knüpft ideell an Vorgänger an. Auf Stein berief sich etwa Wilhelm Hasbach, der an der CAU einen Lehrstuhl für »Wirtschaftliche Staatswissenschaften « innehatte. Doch ob man Hasbach auch als Politikwissenschaftler bezeichnen mag, hänge - so Christian Patz - letztlich davon ab, »ob und wie sehr man Demokrat sein muss, um Politikwissenschaft zu betreiben « (230). Eindeutig demokratisch gesinnt war Hermann Heller, den Michael Holldorf als einen »Vater der modernen Politischen Wissenschaft in Deutschland « (255) würdigt. Etwas zurückhaltender ist Carsten SchlüterKnauer bezüglich Ferdinand Tönnies; doch will er ihn nicht den Soziologen überlassen: Dessen »demokratietheoretisch inklusive politische Willens- 
theorie « und sein »kommunikatives Konzept der Politik « (290) zeigten ihn als »Wegbereiter der modernen Politikwissenschaft « (ebd.). Tönnies lehrte auch am Kieler Institut für Weltwirtschaft und Seeverkehr (IfWuS) und bildet so einen Anknüpfungspunkt zum Beitrag von Alexander Wierzock und Sebastian Klauke, die exemplarisch drei junge Forschende mit ihren Dissertationen beleuchten und zeigen wollen, dass die Deutsche Hochschule für Politik (DHfP) in der Weimarer Zeit kein Monopol auf genuin politikwissenschaftliche Forschung hatte, sondern »die spezifische Betrachtung von Politik selbst an einer vergleichsweise kleinen Universität wie Kiel möglich war« (322).

Eine eigene Abteilung mit drei Beiträgen füllt die Nachkriegs-Politologie zu Michael Freund, dem ersten Ordinarius für »Wissenschaft und Geschichte der Politik«. Birte Meinschien gibt einen Überblick zu Freunds Werdegang, seiner Berufung nach Kiel - wesentlich betrieben vom einflussreichen Journalisten und SPD-Politiker Andreas Gayk - und seinem Politikwissenschaftsverständnis mit »spezifisch historische[m] Zugriff" (367). Zwar konstatiert Meinschien, dass Freund »keine wirkmächtige Rolle in der Geschichte der Politikwissenchaft spielte " (368), doch ist seine zeitgenössische Bedeutung als akademischer Lehrer von nachmaligen Hochschulprofessoren und Politikern sowie als Publizist nicht gering zu veranschlagen. Seine Veröffentlichungen und überlieferten persönlichen Schriften lassen Freund in der Diskussion um die erste Generation der Politikwissenschaftler nach 1945 ambivalent erscheinen. In der Weimarer Republik war er SPD-Mitglied gewesen; 1940 trat er dann - erkennbar aus beruflich-opportunistischen Motiven - in die NSDAP ein. Vorangegangen waren 1938 die Habilitation in Freiburg, in deren Gefolge die Nationalsozialisten ihm allerdings die (davon getrennte) Dozentur verwehrten, sowie aus politischen Gründen Entlassungen aus Verlagsanstellungen. Offenbar war auch seine briefliche Anbiederung an die "Parteiamtliche Prüfungsstelle zum Schutz des nationalsozialistischen Schrifttums « im März 1936 ohne Wirkung geblieben. Darin versichert er, »daß ich mich im Dritten Reich bejahend und positiv zum neuen Staat gestellt und ohne aufdringliche Anpassung [...] das Meine zum Aufbauwerk beizutragen mich bemüht habe« (333). So kann man auch das 1944 von ihm herausgegebene Buch Der falsche Sieg. Worte aus Werken von Georges Sorel interpretieren. Philipp Eulenberger zeigt, dass Freund in dieser - später verschwiegenen - Veröffentlichung nicht nur einen ideologisch schillernden Denker in Originalzitaten vorstellt; er tut dies ohne Quellenangaben und macht sich bei der Auswahl Sorels »Flexibilität der Verortung « (384) zunutze. Die antisemitischen Passagen, der »polternde Ton« (371), die tiefen Griffe »in die Kiste der Ressentiments « (ebd.): das alles ist Sorel im Original, ohne kritische Kommentierung zusammengestellt von Freund. An sein wenig rühmliches Verhalten im Nationalsozialismus, auch an sein SorelBuch, wurde er später unsanft erinnert. Catharina Nies beleuchtet in ihrem Beitrag die Institutsbesetzung der Studierenden im Juni 1969, deren erklärtes Ziel es war, »die reaktionäre Politologie Freunds zu zerstören « (395). Freund selbst sah darin verhängnisvolle Anfänge einer revolutionären Bewegung, welche die Wissenschaft erneut unter ideologische Kuratel zu stellen drohe. Wissenschaft, so war er überzeugt, dürfe »nur ein Gesetz über sich an[erkennen] [...]: das ist die Wahrheit « (416). Seine Sicht der biografischen Wahrheit geht nur aus privatem Schriftverkehr hervor. Im Zuge seines Entnazifizierungsverfahrens schrieb er im Januar 1946 an Gayk: »Ich hätte eben 1933 Deutschland verlassen müssen. So habe ich jetzt zu teuer bezahlt [...]. Dabei war doch alles vergeblich: Ein [beruflicher, C. J.] Rausschmiss folgte auf den anderen « (421). Freunds »ambivalentes Verhältnis zum eigenen Verhalten im Nationalsozialismus « (Meinschien, S. 366) wird hier offensichtlich.

Die letzte Abteilung des Bandes behandelt die Kieler Politikwissenschaft nach Freund. Katia Backhaus widmet sich in ihrem instruktiven Beitrag dem Wirken des Lehrstuhl-Nachfolgers Werner Kaltefleiter (1937-1998) und dessen institutsinternem Kleinkrieg gegen Wilfried Röhrich, seit 1979 zweiter Kieler Professor für Politologie. Unterschiedliche Wissenschaftsverständnisse und Kaltefleiters eher konfliktive Persönlichkeitsdisposition waren die tiefer liegenden Ursachen dieses akademischen >Kalten Krieges<, der um 1993 kurzzeitig in eine sheiße Phase eintrat. Kaltefleiter wollte nicht länger die studentischen Leistungsnachweise seines Kollegen anerkennen und sprach sich offen für eine Institutsteilung aus. Der Studienplan war für einige Semester nach Wissenschaftsauffassungen (»empirisch-strukturell«, »historisch-dialektisch «) gegliedert, ehe der »Hah- 
nenkampf « (DIE ZEIT, S. 468) in »wechselseitig ignorante Koexistenz « (466) überging. Backhaus zieht das Fazit, »dass die Geschichte der Kieler Politikwissenschaft in dieser Zeit als eine in mehreren Hinsichten einzigartige beschrieben werden kann « (474). Zur Geschichte dieser Zeit gehört auch die Entwicklung des Lehramtsstudiums, welcher sich der Politikdidaktiker Klaus-Peter Kruber in einem knappen Überblick widmet. 1974 als Studiengang »Wirtschaft/Politik « etabliert, erwies es sich zunächst als »Sackgasse «(477), weil gar kein entsprechendes Schulfach an Gymnasien in Schleswig-Holstein eingerichtet war. Von ähnlicher Kuriosität war anno 1983 die Abspaltung der Deutschen Gesellschaft für Politikwissenschaft (DGfP) von der DVPW: Die großen ideologischen Konflikte schienen vorüber, wortgewaltige Auseinandersetzungen auf Kongressen gehörten der Vergangenheit an. Tobias Bartels untersucht die Gründe für diese Spaltung. Zwar ist der konkrete Bezug zu Kiel unklar, doch bietet sein Beitrag eine verdienstvolle Zusammenfassung der Erkenntnisse zum Thema. Der Streit war nur nachgelagert ein ideologischer, primär ging es um forschungspolitischen Einfluss. Parallelen zur gegenwärtigen Debatte um die Vergangenheit des Faches und ihrer Vertreter erscheinen naheliegend. Die Herausgeber hätten - zum Beispiel - darauf in einem Resümee eingehen können, ebenso wie auf ihre Anfangsthese von der »exemplarischen Entwicklung " der Kieler Politikwissenschaft. Auch die methodologischen Grenzen der personenzentrierten Herangehensweise hätten sie diskutieren können. Doch ein entsprechendes Schlusskapitel existiert leider nicht. Und das skizzenhafte »Fazit « in der Einleitung (56-59) entschädigt nicht dafür. Zwar behaupten die Herausgeber dort, dass »wichtige Aspekte« der Beiträge »in einigen Details auch als Revision [der Fachgeschichtsschreibung] gelten können «, die außerdem »über diesen universitären Standort hinausweisen « (57). Konkret benennen sie drei. Doch Fragezeichen sind angebracht.

Erstens, so Knelangen und Stein, habe das IfWuS gegenüber der DHfP bisher zu wenig Aufmerksamkeit als politologische Forschungsstelle erhalten. Das vermag der Beitrag von Wierzock/ Klauke aber nicht zu belegen, weil sich die Verfasser über weite Strecken exemplarisch drei Doktoranden widmen (304-322). Die Relation der politikwissenschaftlichen Forschung am IfWuS zur DHfP wird nicht erhellt. So bleibt die eingangs formulierte These der Herausgeber unbeantwortet.

Zweitens, so die Herausgeber, müsse das »Narrativ der unbefleckten Gründergeneration « in der bundesdeutschen Politikwissenschaft korrigiert werden (58). Tatsächlich bietet der Band neue Erkenntnisse für eine differenzierte Auseinandersetzung mit dem Politologen Michael Freund. Das ist doppelt erfreulich, weil sich damit auch Vergleichsmaßstäbe ergeben in der emotionalisierten Debatte über Theodor Eschenburg. Tatsächlich wirkte diese wesentlich von einem "Entlarvungsethos (Wilhelm Bleek) angetriebene Auseinandersetzung bisher vornehmlich als Bühne für akademische Schaukämpfe um Deutungshoheit in der Fachgeschichtsschreibung und um Vormachtstellung in der Fachverbandspolitik. Quellenkritische und nicht vorschnell urteilende Beiträge wie die vorliegenden zu Freund sind zu begrüßen. Letzten Endes stellt sich auch mit Blick auf Freund die Frage, ob Wilhelm Bleeks Feststellung (in Die Geschichte der Politikwissenschaft in Deutschland, München 2001) zu korrigieren sei, wonach die westdeutsche Politikwissenschaft nach 1945 wesentlich »von Hochschullehrern getragen [wurde], an deren Resistenz gegen die nationalsozialistische Diktatur und Eintreten für die freiheitliche Demokratie kein Zweifel bestehen konnte, sieht man von Arnold Bergstraesser ab « (ebd., S. 306). Diese Frage ist nicht einfach zu beantworten, weil sie eine Antwort darauf verlangt, wie vehement ein demokratischer Wissenschaftler, Publizist und Intellektueller in der totalitären NS-Diktatur für seine Überzeugung eintreten hätte sollen oder müssen. Dabei werden moralische Dilemmata aufgeworfen, die von der Politikwissenschaft allein nicht zu beantworten sind. Bei Freund geht es, wie Catharina Nies richtig bemerkt, nicht nur um sein Tun und Lassen als Wissenschaftler und Publizist, sondern auch (wie übrigens bei Eschenburg) sein Handeln als mehrfacher Kindesvater und wirtschaftlicher Versorger einer Familie.

Drittens, so Stein und Knelangen, sei »in Kiel der enge Bezug zur praktischen Politik« (58) seit dem 19. Jahrhundert eine Konstante, die eine mutuelle Charakteristik auszeichne - Ermöglichung politikwissenschaftlicher Forschung dank politischer Entscheidungen einerseits; politologisch geschulte, in Politik und politiknahe Arbeitsbereiche strebende Dozenten und Absolventen andererseits. $\mathrm{Ob}$ wir es hier mit einer Kieler Besonderheit 
zu tun haben, könnte ein systematischer Vergleich mit anderen Standorten zeigen. (Schon die Grenzlage mit der lange Zeit schwelenden SchleswigHolsteinischen Frage, die etwa Falck oder Dahlmann umtrieb, deutet auf eine Sonderstellung. Und in Bezug auf die Bundesrepublik erscheint denkbar, dass die hohe Zahl in Kiel politologisch geschulter Politiker deshalb besonders ausgeprägt ist, weil die CAU als einzige Hochschule Schleswig-Holsteins über ein vollständiges Fachangebot verfügt.) Den Vergleich - eine klassische politikwissenschaftliche Methode - vermisst der Rezensent (auch) in diesem Zusammenhang.

So weist dieser Sammelband zahlreiche Einzelbeiträge mit interessanten und inspirierenden Erkenntnissen auf, die für die Gelehrten- und Ideengeschichte über Kiel hinaus wertvoll sind und vielfältige, disziplinenübergreifende Forschungsanstöße geben können. Die Beiträge über Freund erweisen sich zudem als ergiebig für die gegenwärtige Debatte über die Gründungsprofessoren des Faches in der Bundesrepublik. Und schließlich liefern die Beiträge der letzten Abteilung stimulierende Gedanken zum Verhältnis von akademischer Konkurrenz und ideologischem Positionsdenken, von sozialwissenschaftlicher Forschung, Lehre und Einflussstreben. Deshalb ist dieses Buch auch für die Wissenschaftssoziologie relevant. Doch all dies kann nicht verdecken, dass eine Schlussbetrachtung fehlt. Es wäre fruchtbar und lohnenswert gewesen, die eingangs formulierten Thesen im Lichte der Beiträge zu prüfen und gegebenenfalls zu korrigieren, offene Fragen zu formulieren sowie den Standort Kiel systematisch in den Zusammenhang der bundesdeutschen Politikwissenschaft einzuordnen.

\section{Clemens Jesenitschnig}

Mark SCHWEDA: Entzweiung und Kompensation. Joachim Ritters philosophische Theorie der modernen Welt, Freiburg/München: Karl Alber, 2013, 480 S., $49 €$

Die Philosophie Hegels - im Besonderen seine Rechtsphilosophie - bedeute im Kern eine Auseinandersetzung mit der Französischen Revolution, ja, es gebe »keine zweite Philosophie, die so sehr und bis in ihre innersten Antriebe hinein Philosophie der Revolution« sei wie eben jene Hegels. Diesen Gedanken brachte Joachim Ritter 1957 in seinem Aufsatz Hegel und die französische Revolution auf den Punkt, und damit trug er entscheidend zur Renaissance einer liberalen Hegel-Lektüre in Deutschland bei, die Hegel von dem Verdacht entlastete, Apologet eines reaktionären preußischen Obrigkeitsstaat gewesen zu sein, andererseits aber auch die Wege seiner linkshegelianischen Vereinnahmung umging. Dieser Beitrag zur Wiederbelebung einer »Hegelschen Mitte (Henning Ottmann) wird Joachim Ritter gemeinhin ebenso zugebilligt wie andererseits, durch seine Aristoteles-Interpretation, sein Beitrag zu einer »Rehabilitierung der praktischen Philosophie« so der Titel des einschlägigen, von Manfred Riedel herausgegebenen Sammelbandes - in der Nachkriegsbundesrepublik. Dass im Hintergrund dieser im einzelnen durchaus wirkungsreichen Forschungsbeiträge Ritters allerdings ein übergreifendes, nicht weniger als ambitioniert zu nennendes philosophisches Anliegen stand, wurde in der Rezeption - und zwar in der Abgrenzung von Ritters Überlegungen nicht weniger als in ihrer Aufnahme - häufig eher verstellt. Auch das Fehlen eines systematischen Hauptwerkes Ritters, der seit 1946 in Münster lehrte und dort mehrere Generationen teils später namhafter akademischer Schüler um sich scharte, dürfte einiges zu dieser eher selektiven Wirkungsgeschichte beigetragen haben.

Der Göttinger Philosoph Mark Schweda hat sich nun jedoch in seiner gründlichen Studie Entzweiung und Kompensation, hervorgegangen aus einer von Volker Gerhardt betreuten Dissertation, Joachim Ritters philosophischer Theorie der modernen Welt angenommen und diese aus verschiedenen Aufsätzen Ritters, teils auch aus unveröffentlichtem Nachlass-Material, rekonstruiert. Das Ergebnis macht für Ritter zu Recht einen wichtigen Beitrag zur deutschen Nachkriegsphilosophie geltend, den es in seinen unausgeschöpften Potentialen und angesichts mannigfaltiger Verzerrungen und Missverständnisse erst noch zu bergen gelte. Ritters philosophisches Anliegen gewissermaßen unter den Vorbehalten hervorzuziehen, die es immer noch überlagern, ist das Anliegen des Autors. Seine Arbeit reiht sich damit zum einen ein unter zahlreiche jüngere Forschungsbeiträge zur politisch-philosophischen Ideengeschichte der Bundesrepublik, in deren Zusammenhang auch Joachim Ritter bereits in den Blick genommen wurde, nicht zuletzt freilich mit Fokus auf einen über seine Schüler wie Hermann Lübbe, Odo Marquard 
und Robert Spaemann vermittelten Beitrag zu einer »liberalkonservativen Begründung der Bundesrepublik«(Jens Hacke).

Auch Schweda hebt ein in diesem Sinne liberales oder liberalkonservatives Verständnis der Ritterschen Philosophie hervor, das es insbesondere gegen den von Jürgen Habermas und anderen erhobenen Vorwurf eines sich bei Ritter angeblich abzeichnenden Neokonservatismus zu verteidigen gilt. Angesichts des immer noch problematisierungsbedürftigen Schlagworts einer Ritter-Schule, hinter dem Ritters philosophisches Denken selbst eher verdunkelt wurde und wird, ist zum anderen ebenso hervorzuheben, dass Schweda Ritters Philosophie auch gegenüber ihrer differenzierten und eigenwilligen Aufnahme im Kreise seiner Schüler als eigenständig hervorhebt. Dies ist zweifellos eines der größten Verdienste dieser Arbeit, die das Denken Ritters profund rekonstruiert. Den Fluchtpunkt dieses philosophischen Ansatzes, und zwar in jener Gestalt, die Ritters Münsteraner Studierenden in den fünfziger und sechziger Jahren begegnen konnte, bildet eine an Hegel, Aristoteles und den Vorsokratikern geschulte »Hermeneutik geschichtlicher Wirklichkeit«. In dieser verbindet Joachim Ritter eine philosophische Zeitdiagnostik, welche den Stand eines in der modernen Welt erreichten wissenschaftlich-technischen, aber auch politischen und allgemein gesellschaftlichen Fortschritts rückhaltlos anerkennt, mit einem von Hegel her zu verstehenden geschichtsphilosophischen Fundament und einem philosophischen Selbstverständnis, das nicht weniger als den Anspruch erhebt, die eigene Zeit auf den Begriff zu bringen.

Da Aristoteles und Hegel spätestens ab den 1950er Jahren die beiden zentralen philosophischen Referenzautoren Ritters sind, kann es nicht überraschen, dass Schwedas in drei großen Schritten ausgearbeitete Rekonstruktion insbesondere von Ritters Hegel- und Aristoteles-Interpretationen ausgeht. Ersterer wird dabei insbesondere in Ritters Auseinandersetzung mit dessen Rechtsphilosophie wirksam, mittels derer Ritter ein Verständnis der modernen Welt entwickelt, die durch eine Entzweiung von bürgerlicher Gesellschaft und Subjektivität bestimmt sei. Dabei ist für Ritter vor dem Hintergrund der noch jungen Bundesrepublik mit ihren historischen Belastungen bei gleichzeitiger wirtschaftlicher Prosperität eine als gefährdet und gefahrvoll zugleich wahrgenomme- ne moderne Lebenswirklichkeit philosophischer Bezugspunkt, die auf Grundlage sehr unterschiedlicher weltanschaulicher und theoretischer Standpunkte infrage gestellt wird. Diese von Ritter als fortschritts- bzw. verfallstheoretisch apostrophierten Tendenzen, vertreten von revolutionären wie reaktionären Geistern, Konservativen und Marxisten, Existentialisten und Romantikern, logischen Positivisten und christlichen Abendländlern, opponieren jeweils gegen ein wahlweise als unvollendet oder dekadent empfundenes Zeitalter. Diese Beobachtung veranlasst Ritter, seine Gegenwart mithilfe einer Auslegung der Hegelschen Rechtsphilosophie auf den sachlichen Kern dieses Problems hin zu befragen, und er gelangt so zu einer Interpretation dieser modernen Welt, in deren Zentrum der Begriff der Entzweiung steht. Diese beschreibt in Ritters Verständnis eine moderne Lebenswirklichkeit, in der die Sphären der bürgerlichen Gesellschaft und der individuellen Subjektivität unvereinbar in Gegensatz zueinander getreten zu sein scheinen. Einerseits löst sich die bürgerliche Gesellschaft im Ganzen aus vormals sie übergreifenden Bindungen; sie tritt auf als eine gleichsam geschichtslose Gesellschaft, deren technische und wissenschaftliche Entwicklung sich allein an der Bedürfnisnatur des Menschen und ihrer Befriedigung orientiert. Andererseits verbleiben die einzelnen Menschen auch unter diesen Bedingungen in einem Netz tradierter Bindungen situiert, sind aber zugleich freigesetzt, autonom über diese zu bestimmen. Ritter akzeptiert diese Beschreibung eines Auseinandertretens von Zukunft und Herkunft als Charakterisierung der modernen Welt. Mehr noch aber weist er die Suggestion von Fortschritts- und Verfallstheoretikern zurück, dass diese moderne Welt einen Bruch mit der abendländischen Tradition bedeute, sondern er begreift die Struktur der Entzweiung auf einer höheren, geschichtsphilosophischen Ebene ihrerseits als Ausdruck einer geschichtlichen Kontinuität. Nach der griechischen Polis und dem Christentum aktualisiere sich in der Französischen Revolution die Bestimmung des Menschen zu Freiheit und Vernunft in einer weiteren Entwicklungsstufe, die das Prinzip einer universell zu verstehenden menschlichen Freiheit zur Grundlage jeder politischen Ordnung erhebt. Das Auseinandertreten von Zukunft und Herkunft in der Moderne bedeutet für Ritter nichts anderes als einen weiteren Schritt im weltgeschichtlichen Prozess humaner Freiheit. 
Mit diesem Anliegen einer zeitgemäßen Aktualisierung Hegels einher geht bei Ritter ab den 1950er Jahren die Hinwendung zu einem Verständnis praktischer Philosophie, das er von Aristoteles gewinnt. Der für die moderne Welt charakteristischen Entzweiung von Subjektivität und Gesellschaft korrespondiere nämlich eine Auflösung der auf Aristoteles zurückreichenden Pbilosophia practica universalis im Laufe der Neuzeit. Hier seien ein Normen postulierender moralphilosophischer Idealismus einerseits und andererseits die deskriptiv orientierten Sozialwissenschaften auseinandergefallen. Zuletzt habe noch Hegel versucht, die aristotelische praktische Philosophie in seiner Zeit philosophisch zu vergegenwärtigen, »um aus dem Zusammenhang der modernen politischen Wirklichkeit zum Begriff hervorzubringen, was ohne ihre Vergegenwärtigung nicht ausgesagt und nicht begriffen werden kann.« Mithin ist Ritter in Bezug auf Aristoteles wie im Falle der Hegelschen Rechtsphilosophie darum zu tun, ein aristotelisches Verständnis praktischer Philosophie und bürgerlichen Lebens auf der Höhe seiner Zeit zu aktualisieren.

Mit seiner Interpretation der klassischen Autoren - auch der Vorsokratiker, wie Schweda in einem weiteren Kapitel darlegt - reagiert Ritter auf wahrgenommene Herausforderungen seiner Zeit. Und er folgt der von ihm dabei zugleich formulierten Aufgabe der Philosophie, innerhalb der Gemeinschaft freier Bürger und auf vernünftigen Gründen basierend eine in der menschlichen Praxis bereits erschlossene Welt reflexiv zu vergegenwärtigen. Diese »Hermeneutik der geschichtlichen Wirklichkeit« bringt Ritters Philosophieverständnis und mithin auch dessen Selbstbild auf den Punkt: Die Philosophie thematisiere »in reflexiver Rückwendung das umfassende Weltverständnis, das der gesellschaftlich-politischen Praxis ibrer eigenen Zeit zu Grunde liegt« (369).

Im Rahmen der entsprechenden Rekonstruktion der Ritterschen Deutung der modernen Welt weist Schweda auch den immer wieder gegen Ritter erhobenen Vorwurf eines Neokonservatismus zurück. Denn weit davon entfernt, vormoderne ethische und politische Ordnungsvorstellungen in die Gegenwart übertragen zu wollen, dient Ritter der Rückgriff auf Aristoteles und Hegel zur Vergewisserung über die Aufgabe der Philosophie, in jeder Epoche der Geschichte stets aufs Neue diesen in der schier unüberblickbaren Vielfalt menschlicher Wirklichkeit sich abzeichnenden Grund von Vernunft und Freiheit auszuweisen. Die in der Moderne erreichte Autonomie und politische Freiheit des Menschen ist für Ritter daher unhintergehbar. Die von Habermas und anderen erhobene Kritik übersieht und verfehlt insofern den entscheidenden Punkt der Ritterschen Philosophie, zeigt dadurch aber auch, wie sehr Ritters eigenes philosophisches Anliegen auch gegenüber Vereinnahmungen und Vereinseitigungen seitens seiner Schüler hervorzuheben ist. In der Vergangenheit haben wechselseitige Polemiken hier nicht nur einen unvoreingenommenen Blick auf Ritters Philosophie erschwert, sondern sie mit Verkürzungen und Missverständnissen geradezu verstellt. Andererseits kann es angesichts der anspruchsvollen metaphysischen und geschichtsphilosophischen Voraussetzungen des Ritterschen Projekts auch nicht erstaunen, dass einige seiner Schüler es gerade um diese Prämissen gekürzt haben, wie Schweda es in Auseinandersetzung mit der Aufnahme des Ritterschen Kompensationstheorems bei Hermann Lübbe und Odo Marquard zeigt. Der Rezeption Ritters bei seinen drei wohl prominentesten akademischen Schülern - neben Lübbe und Marquard tritt da auch noch Robert Spaemann - ist nämlich ein letztes größeres Kapitel von Schwedas Arbeit gewidmet, in dem gerade die manifesten Differenzen gegenüber Ritter wie auch untereinander im Mittelpunkt stehen.

Bei allem Festhalten an dem in der modernen Welt erreichten Stand menschlicher Freiheit sind es gerade auch die der Moderne innewohnenden Ambivalenzen und Unselbstverständlichkeiten, ihre mit der Entzweiung einhergehenden Gefährdungen, welche Ritter ausweist. Denn indem durch die Entzweiung die bürgerliche Gesellschaft sich aus vormals vorgegebenen Bindungen emanzipiert und die Individuen zu selbstbestimmter Autonomie freisetzt, verzichtet sie zugleich auf eine positive Bestimmung menschlicher Freiheit. Diese ist allein auf Ebene der Subjektivität möglich und doch zugleich notwendig, um die Überzeugung, »daß mit dem Menschenrecht der modernen Gesellschaft alle Menschen frei werden«, überhaupt positiv bestimmen zu können. Schweda hebt hier gegen den Einwand, Ritter vermeide es im Gegensatz zu Hegel eine die Entzweiungsstruktur versöhnende Komponente einzuführen, die Bedeutung der Subjektivität hervor. Allein in ihrer Sphäre sei Versöhnung möglich. Somit wird das 
einzelne Individuum zum Träger der Aufgabe der Versöhnung. Auch der Staat ist allenfalls in der Lage, geeignete Rahmenbedingungen zu schaffen, um das fragile Gleichgewicht von bürgerlicher Gesellschaft und Subjektivität im Rahmen der Entzweiung zu erhalten. Mithin »bleibt die vollständige Verwirklichung des Menschseins in der Moderne letztlich auf die einzelnen Individuen selbst und ihre Bereitschaft verwiesen, sich im Rahmen ihres Lebens in der modernen bürgerlichen Gesellschaft den von dieser freigegebenen Überlieferungsbeständen zuzuwenden und sie sich zu eigen zu machen, um auf diesem Weg die Vermittlung von Herkunft und Zukunft im konkreten Einzelfall zu vollziehen.« (193)

Schwedas Arbeit eröffnet einen Zugang zu einem philosophischen Grundanliegen Ritters, das aus seinen verstreuten Publikationen erst systematisch herausgearbeitet werden musste. Für diese Leistung wurde sie unter anderem mit WolfErich-Kellner-Preis der Friedrich-Naumann-Stiftung sowie mit dem Karl-Alber-Preis 2014 ausgezeichnet. Es bleiben dennoch wichtige Fragen für weitere Forschungsarbeit offen. $\mathrm{Da}$ ist zum einen Ritters Biografie, die Schweda zwar in einem Abriss von Leben und Werk auch mit Blick auf die Brüche und Kontinuitäten des Ritterschen Denkweges darstellt. Dennoch bleibt die Frage im Raume, was diesen Joachim Ritter, der in der Weimarer Republik mit dem Marxismus sympathisierte, der sich dann aus Opportunitätsgründen mehr oder weniger stark auf die Nationalsozialisten einließ und 1946 schließlich mit deutlich kulturpessimistisch gefärbten Tönen aus der Kriegsgefangenschaft zurückkehrte, um seinen Lehrstuhl in Münster anzutreten, eigentlich selbst mit der modernen Welt und ihren Zumutungen versöhnt hat. Der Joachim Ritter, den Schwedas Arbeit seinen Lesern vorstellt, ist der Ritter auf dem Höhepunkt seines philosophischen Werkes, in den Jahren zwischen seiner Rückkehr von einem Auslandsaufenthalt in der Türkei 1955 und seiner Emeritierung im Jahr 1968. Ritters vortürkische Philosophie, um eine Prägung Odo Marquards aufzugreifen, die aber seine ersten Münsteraner Studenten sehr geprägt haben dürfte, bleibt hier etwas außen vor. Daneben bleibt auch eine weitere wissenschaftliche Erschließung des sich im Deutschen Literaturarchiv Marbach befindlichen Nachlasses Ritters zu wünschen, den auch Schweda in seiner Arbeit an einigen Punkten - insbesondere was Ritters
Rezeption der Vorsokratiker angeht - berücksichtigt.

Eine ganz andere Frage wiederum ist es, ob und wie es möglich sein kann, Ritters Anliegen, das in der Entzweiung Auseinandertretende im Medium der Philosophie in seiner Zusammengehörigkeit $\mathrm{zu}$ begreifen, jenseits eines reinen Postulats auch vierzig Jahre nach seinem Tod wieder produktiv aufzugreifen. Diese Frage eröffnet sich durch die von Schweda entwickelte Gesamtsicht auf Ritters Denken, und doch bleibt die Befürchtung, dass sie eine Forderung aufwirft, die in unserer Gegenwart mehr denn je unerfüllt bleiben muss. Auch Ritter selbst hat diesen Ansatz, das Entzweite in seiner ursprünglichen Zusammengehörigkeit auch in Zeiten gesellschaftlicher Differenzierungen, die es zugleich nicht zu verwerfen gilt, festzuhalten, nicht hinreichend detailliert ausgeführt. Offen ist auch die Frage, ob eine Vergegenwärtigung des Ganzen in seiner Entzweiung im Sinne Ritters in den Augen derjenigen, denen eine wie auch immer herzustellende oder wiederherzustellende Einheit im Grunde lieber wäre, jemals überzeugend auftreten könnte. Und die Begründungslasten, die ein solches Projekt zu schultern hätte, dürften in der Zwischenzeit nicht geschwunden sein, sodass sich womöglich gerade ein pragmatischeres Verhältnis zur modernen Welt im Sinne Lübbes und Marquards, das die Aufgabe, deren weltgeschichtlichen Zusammenhang philosophisch zu rechtfertigen, für unnötig oder obsolet hält, als Alternative aufdrängt.

Martin Ingenfeld

Peter SLOTERDIJK: Die schrecklichen Kinder der Neuzeit, Berlin: Subrkamp, 2014, 489 S., $26,95 €$

Bei aller neuzeitlichen Bejahung der Freiheit und der neuzeitlichen Errungenschaften der Revolution sieht Sloterdijk in den jüngsten europäischen aber auch globalen - Entwicklungen Gefahren des Kulturbruchs und des Untergangs. Die Bedürfnisse werden immer größer als die Möglichkeiten, sie zu erfüllen. Die vor allem in den Politikwissenschaften herbeigeschriebene Harmonie von Menschenrechten und globaler Herrschaft werden von Sloterdijk als »Phantasien« (93) entlarvt. Ebenso interessant wie brisant ist, dass er dem Begriff der Erbsünde durchaus Realitätsgehalt zuspricht, 
wenn der Henker Ludwigs XVI. einen Zustand der völligen Rechtsabwesenheit eröffnet und dieser einer politischen Wiederholung der Erbsünde gleichkommt (103).

Im Begriff der »Filiation « verwendet Sloterdijk die Erbsünde in einem säkularen Sinne, ohne allerdings einen rationalen Grund dafür angeben zu können; diese Art Erbsünde muss ebenso geglaubt werden wie ihr christliches Verständnis, welches allerdings, gleich eingangs, zusammen mit der Kritik an Augustinus, verworfen wird. Sloterdijk reformuliert »religiös codierte "Sachgehalte in weltlichen Ausdrücken, weil - wie er behauptet - »dem zeitgenössischen Denken der Zugang zum Konzept der Erbsünde nicht mehr offen steht « (27). Statt »Filiationen «, Weitergaben des Ethos und der Traditionen, sieht Sloterdijk das Vorhandensein eines revolutionären Hiatus, der sich von der Französischen über die Russische Revolution permanent vergrößert.

Mit einer Reihe von Beobachtungen will Sloterdijk diese bedrohliche Abwärtstendenz belegen. $\mathrm{Da}$ ist zunächst die hübsche Geschichte des Aufstiegs der Madame Pompadour, die 1757 bei einem abendlichen Fest, als die Nachricht von der Niederlage der französischen Truppen in der Schlacht bei Roßbach gegen die zahlenmäßig unterlegene Streitmacht Friedrichs II. von Preußen eintraf, ausrief: "apres nous le déluge", "nach uns die Sintflut « (31 ff.). Ihr Fehler war freilich, nicht zu sehen, dass der Untergang schon zu ihrer Zeit begonnen hatte.

Mit eindringlichen Rückgriffen auf Ereignisse der Französischen und Russischen Revolution, auf Aussagen Napoleons (95-178) und der Rede Himmlers 1943 vor SS-Leuten in Posen (182), zeigt Sloterdijk, wie auf der einen Seite das »Monstrum « - in Anlehnung an die Vorstellung von den Monstren der griechischen Urzeit - in der Geschichte auftaucht und wie andererseits wieder die entsprechenden Reichsträume schnell in sich zerfallen sind. Wenn Sloterdijk in Anspielung auf die Französische Revolution davon spricht, wie »die Anreger Exekutoren und Interpreten der Blutbäder immerzu von Freiheit und Gleichheit, von Eigentum und Fortschritt, von Menschenrecht, Verfassung und Herrschaft der Vernunft reden« (59), hätte man doch gerne ein Urteil über das, was nun, summa summarum, aus den Intentionen von $\mathrm{Ma}$ rat und Robespierre geworden sei. Oder, statt im europäischen Haus eine monströse Baustelle zu sehen, wären Hinweise auf das friedvolle Zusammenwirken von Nationen, die sich früher bekriegt haben, im Rahmen der Europäischen Union realistischer gewesen. Demokratie als Botschaft der Freiheit und hoffnungsvoller Entwicklung - wie sie Hannah Arendt in den USA ausgemacht hat geht im Wirbel düsterer Beobachtungen unter.

Sloterdijk thematisiert die Entzweiung von Herkunft und Zukunft, die Joachim Ritter mit den geschichtlichen Folgen von Französischer Revolution und ungebremster kapitalistischer Entwicklung heraufkommen sah. Aber während Ritter sich an die freiheitlichen Aspekte der hegelschen Dialektik (das Bewusstwerden der Freiheit) hielt, denkt Sloterdijk an die Hegelsche Schlachtbank der Geschichte. Dabei sieht er die aktuelle geschichtliche Lage eher mit Nietzsche in einer Haltlosigkeit und scheinbaren Richtungslosigkeit, in einem chronischen Nach-vorne-Stürzen (71). Der Sturz ins Bodenlose, der bei Nietzsche trotz des Fehlens eines rettenden Gottes in einer Befreiung resultiert, wird bei Sloterdijk zum Sturz in einen geschichtlichen Abgrund. Der für Viele gefährliche und allzu weit geöffnete Hiatus zwischen Tradition und Zukunft, den Sloterdijk immer wieder beschreibt, erinnert an die Untergangsbefürchtungen von Spengler. So ganz losgelöst von der in den USA ausgelösten demokratischen Freiheitsdynamik wird man die europäische Entwicklung nicht sehen können. Es ist einigermaßen schwierig zu erkennen, warum Sloterdijk einen Abschnitt »Der Bastard Gottes « einfügt, mit dem er zentral das christliche Geheimnis der Menschwerdung angreift (278-311). Der Grund ist wohl die naturalistische Orientierung am Generationenprozess, mit dem Vorwurf, dass das Neue Testament antigenealogische Impulse zur Folge gehabt habe (486). Wenn es Sloterdijk wirklich um Inklusion des Exkludierten (so auf S. 484) ginge, müsste er doch das christliche Liebesethos würdigen. Die Schlüsselstellung des Prinzips der genealogischen Kontinuität - hätte man früher nicht vom Gesetz des Blutes gesprochen? - reicht nicht aus, um ethische Prinzipien der Besonnenheit und Gerechtigkeit abzuleiten, um - wie Sloterdijk beklagt, die "Asymmetrie zwischen Vergangenheitsbewusstsein und Zukunftserwartung, die ein unlebbares Ausmaß erreicht hat « (485) zu schließen.

Nach der Lektüre dieses Buches sollte man zur Erleichterung des Gemüts zu Jeremy Rifkins Die Null-Grenzkosten-Gesellschaft greifen, das, frei 
von Schwarzmalerei und Salon-Pessimismus, positive Lösungen für kritische Entwicklungen des Kapitalismus anbietet.

\section{Karl-Heinz Nusser}

\section{Werner WEIDENFELD: Europa. Eine Strategie;} München: Kösel, 2014, 128 S., 12,00 €

Strategen sind in Zeiten der europäischen Finanzkrise besonders gefragt. Der Autor, emeritierter Professor für Politische Systeme und Europäische Einigung an der Ludwig-Maximilians-Universität München und Direktor des Zentrums für angewandte Politikforschung, setzt mit einer $\mathrm{Zu}$ standsbeschreibung ein, in der Vertrauensverluste und Krisenmeldungen überwiegen. Dennoch bewertet er die Integration Europas als eine Erfolgsgeschichte. Warum? Es sind drei persönliche Erlebnisse, die Weidenfeld bewogen haben, an Europas Zukunft in der Bündnisform einer EU zu glauben. Die Schaffung eines stabilen Rahmens der politischen Kultur und eines freiheitlichen Systems nach der Katastrophe des Zweiten Weltkriegs, die Schaffung eines stabilen Binnenmarktes mit finanzpolitischer Absicherung und die sichere vertragliche Regelung der Europäischen Integration. In sechs Kapiteln: Problemlagen, Identität, Legitimation, Führungskultur, Mitverantwortung und Strategie-Idee unternimmt er den Versuch, das Europa-Drama der letzten Jahre als eine Erosion des Politischen zu analysieren. Die größte Gefahr sieht er, bedingt durch die wachsende Kompetenz der EU, in der Auszehrung staatlicher Zuständigkeit, die logischerweise zu einer Entleerung demokratischer Substanz in den einzelnen Nationalstaaten führe. Damit werde der Ort demokratischer Verantwortung immer weniger identifizierbar. Auch die Europapolitik habe im letzten Jahrzehnt vieles eingebüßt. Statt Zuversicht, Gewissheit und Kalkulierbarkeit dominierten Hektik und strategische Konfusion, was zu einer machtpolitischen Unsicherheit führe. In dieser Phase wird auch eine europäische Identität in Frage gestellt, Weidenfeld spricht von einem »vagabundierenden Identitätsbedürfnis«. Deshalb müsse man kulturelle Muster der neuen Epoche entwerfen. Dazu gehörten die Identifizierung mit dem Wahlakt zum Europäischen Parlament oder eine lebhafte öffentliche Auseinandersetzung mit europäischer Politik. Doch leider bilde Europa ge- genwärtig nur einen artifiziellen Nebenschauplatz, was unter anderen in dem Scheitern der Referenden in Frankreich und in den Niederlanden zum Ausdruck käme.

Auf der Suche nach den Ursachen dieser Phänomene verweist der Autor auf ein fehlendes operatives Zentrum in Europa und ein Defizit an strategischem Denken. Aus diesem Grund regt er eine Neugründung des europäischen Integrationsmodells an. Weidenfelds Vorschläge zielen dabei auf die Stärkung der globalen Rolle Europas, wobei die Abschottung der nationalen von der europäischen Ebene im politischen Diskurs aufgehoben werden müsse. Das betreffe auch die Handlungsmodelle der Deutschen, deren Sonderbewusstsein im 19. und 20. Jahrhundert in Europa unermesslichen Schaden angerichtet habe.

Eines der komplexesten Probleme im heutigen Europa ist der Legitimationsanspruch der Europäischen Union, in der »weder über das Europäische Parlament noch über die nationalen Regierungen und die nationalen Parlamente ... ein europäischer Zusammenhang der Rechtfertigung und Akzeptanz von Politik vermittelt (wird) «. Die Reihe dringender Maßnahmen ist lang. Einige Beispiele verdeutlichen das: die Schaffung europäischer Medienstrukturen für eine europäische Öffentlichkeit, die Schaffung eines eigenen Parlaments der Euro-Länder, die Initiierung europäischer Bürgerinitiativen, um Europa von unten erlebbar zu machen.

Zwei weitere Problembereiche nennt Weidenfeld, um die unausgeglichene Rolle Europas unter den Weltmächten zu charakterisieren: die fehlende Führungskultur und die notwendige Verteilung der Mitverantwortung auf alle Mitglieder der EU. Im ersteren Fall gehe es um die gemeinsam mit den USA schwindende Rolle Europas unter den globalen Mächten. Da auch die transatlantischen Beziehungen unter den amerikanischen Geheimdienstüberwachungen seitens der NSA leiden, habe sich in der Zwischenzeit zwischen beiden Mächten ein tiefes Misstrauen herausgebildet, das sich erst im Zuge gravierender Veränderungen der Außen- und Sicherheitspolitik auflösen werde. Im zweiten Fall gelte es den Mangel an europäischer Identität in den Nationalstaaten zu überwinden. Deshalb müsse man neue Modelle einer Integrität entwickeln, die gegenwärtig daran scheitern, dass die Spannungen zwischen den einzelnen Funktionsträgern der EU und deren Intransparenz in den 
einzelnen Mitgliedstaten der EU zu Distanz gegenüber Brüssel und zur Flucht in Populismus führe.

Die stellenweise äußerst komplexe Argumentation weist dennoch transparente Lösungsvorschläge auf. Die Bürger/innen in den einzelnen Mitgliedsstaaten der EU müssten begreifen, dass ihre Partizipation an Entscheidungsprozessen für die Zukunft unseres Kontinents entscheidend sei. Und die Lösung? Eine Kommission zum Thema Dezentralisierung und Rückentwicklung von Kompetenzen müsse eingesetzt werden, denn die Partizipation an den politischen Prozessen könne sich beispielsweise nicht in den Wahlen zum Europäischen Parlament erschöpfen. Vielmehr sei die Schaffung einer Partizipationskultur notwendig, um eine wie auch immer strukturierte europäische Öffentlichkeit herzustellen.

Die der strategischen Streitschrift für Europa beigefügten Literaturhinweise verweisen auf eine Vielzahl von Positionen, unter denen ein breites Spektrum an etablierten deutschen akademischen Meinungsträgern vorherrscht. Was fehlt, sind sowohl die westeuropäischen kritischen Stimmen als auch ein differenziertes Positionsspektrum süd-, ostmittel- und südosteuropäischer Meinungen zur gegenwärtige Krisensituation in Europa.

\section{Wolfgang Schlott}

Helmut WILLKE: Regieren. Politische Steuerung komplexer Gesellschaften, Wiesbaden: Springer VS, 2014, 154 S., $€ 17,99$

Dieses Buch handelt vom Regieren, es handelt von politischer Steuerung und es handelt von Systemsteuerung. Aus einem strengen systemtheoretischen Blickwinkel analysiert Helmut Willke die Voraussetzungen und Folgen politischer Steuerung.

Der vorliegende Band behandelt nicht das institutionelle Zusammenspiel von Regierung, Parlament und Parteien. Hier liegt beileibe keine Kurzfassung einer einführenden Regierungslehre vor. Diskutiert wird die Steuerungsfähigkeit des politisch-administrativen Systems im Zeitalter hoher gesellschaftlicher Komplexität. Über weite Strecken liest sich der Text wie eine Einführung in die Systemtheorie. Dabei greift Willke auf die zentralen Arbeiten von Niklas Luhmann zurück sowie auf Entwicklungen der Systemtheorie, die der
Autor in den zurückliegenden Jahrzehnten selbst mit geprägt hat. Seinen Blick schärft er immer wieder in Abgrenzung zu konkurrierenden Konzeptionen des Regierens, die beispielhaft von Fritz Scharpf, Renate Mayntz, Aaron Wildavsky oder Herbert Simon vertreten werden.

Jede Form von Regierungshandeln ist in erster Linie Steuerungshandeln; es geht mithin um die "politische Steuerung komplexer Gesellschaften « (2). Zunächst findet politische Steuerung als beabsichtige Beeinflussung einzelner gesellschaftlicher Systeme statt. Doch in ihrer selbstreferentiellen, operativ geschlossenen Wirkungsweise sind die Systeme allenfalls in der Lage, sich selbst zu steuern. Willkes »steuerungsskeptische Perspektive" (5) führt zu der begründeten Annahme, dass erfolgreiche Eingriffe in einzelne Politikfelder nur äußerst selten gelingen. Mit anderen Worten: Jede Beschäftigung mit Regierungshandeln als Steuerungshandeln »sieht sich heute mit einem Trümmerhaufen gescheiterter praktischer Steuerungsvorhaben und Steuerungshoffnungen konfrontiert« (3).

Moderne Gesellschaften erscheinen als hochkomplexe Gebilde und sind ihrem Wesen nach funktionell differenziert. Dies sind Systeme, »die selbstreferenziell auf sich selbst Bezug nehmen, sich durch spezifische Bedeutungen abschotten und sich am Ende zu operativ geschlossenen Kommunikationssystemen verdichten « (47). Gesellschaft besteht damit als Ordnung aus Differenzen; es sind sogenannte Leitdifferenzen, die als binäre Codes verschlüsselt sind. Das politische System ist demnach durch Macht-Ohnmacht codiert, die Wirtschaft durch Zahlung-Nichtzahlung. Politische Interventionen in andere Systeme bleiben damit beinahe wirkungslos; bestenfalls werden Resultate sichtbar, die nicht beabsichtigt waren. Andererseits findet politische Steuerung statt, gesellschaftliche Systeme stehen immer wieder unter dem Einfluss von Politik und somit rückt die »Frage nach den möglichen Formen der geordneten Verschränkung von operativer Geschlossenheit und externer Anregung « (36) in den Vordergrund. Unter der Bedingung struktureller Kopplung sind unterschiedliche Systeme in der Lage, zu kommunizieren: So sind zum Beispiel Politik und Recht über die Verfassung verbunden; Recht und Wirtschaft stehen durch die Institutionen von Eigentum und Vertrag in Verbindung. Was bei Niklas Luhmann unter struktureller Kopplung firmiert, bezeichnet Willke als Kontextsteuerung. 
Angesichts einer Fülle komplexer, selbstreferentieller Systeme begreift Willke Regieren als das "Management systemischer Kontingenzen" (57). Organisieren und steuern sich diese Systeme hingegen selbst, tritt die Politik gleichsam automatisch in den Hintergrund. Diesen Zusammenhang der in Teilen obsoleten Politik hat Willke in den 90er Jahren als »Ironie des Staates « beschrieben.

Jeder Teilbereich moderner Gesellschaften funktioniert nach je eigenen, spezialisierten Steuerungsregeln. Kommt das System Politik in seiner herausgehobenen Funktion wieder ins Spiel, dann besteht seine Aufgabe darin, »als einziges System für das Ganze von Gesellschaft zuständig zu sein, weil es seine Funktion ist, kollektiv verbindliche Parameter der Steuerung zu definieren « (65/66). In der Verbindung systemischer Selbststeuerung und Kontextsteuerung zur Überwindung operativer Geschlossenheit weist Willke der Politik eine gestaltende Rolle zu. In dieser theoretischen Konzeption besteht für Willke Anlass zum Optimismus, denn »dann sehe ich keinen Grund, die Möglichkeit politischer Steuerung zu bestreiten« (67).

Prozesse der Globalisierung führen dazu, dass Politik »grenzenlos«, »ortlos « und »de-nationalisiert« erscheint (69). Weder politische Steuerung, noch Selbststeuerung von Problemlagen in globalem Maßstab sind in Sichtweite. Doch immerhin gibt es die »verschiedensten Formen dezentraler, funktionsspezifischer Selbststeuerung, in Gebilden «, die Willke »laterale Weltsysteme « nennt (86). Diese Systeme, wie z. B. das globale Finanzsystem bilden ihre eigenen globalen Kontexte mit einer spezifischen Kommunikation, streben Möglichkeiten der Selbststeuerung an können »sich dadurch aus der Vormundschaft der Politik emanzipieren « (87). Gerade mit Blick auf die Verwerfungen der globalen Finanzkrise erscheint es mehr als zweifelhaft, ob sich einzelne Teilsysteme der Gesellschaft noch stärker dem Einfluss der Politik entziehen sollten. Die Möglichkeiten und Grenzen politischer Steuerung der Finanzwelt thematisiert Willke als kurze Fallstudie. Allerdings verbleibt vieles als eher nüchterne Feststellung stehen und endet mit der zutreffenden Schlussfolgerung, »dass der Druck zunimmt, zu gemeinsamen, möglichst globalen Lösungen zu kommen, weil die Kosten einer weiteren Katastrophe für alle Betroffenen steigen « (94).

Gelingende Steuerung beruht auf der Verfügbarkeit von Wissen. Das politisch-administrative System steht Politikfeldern gegenüber, die nicht nur eine differenzierte Interessenvielfalt der Gesellschaft widerspiegeln, sondern gleichzeitig in ihrer jeweiligen Operationsweise professionalisiert und hochspezialisiert sind. Während die Politik danach strebt, brauchbares Wissen über diese Regelungsmaterie zu erlangen, stellen komplexe Politiksektoren »für den durchschnittlichen Wahlbürger schwarze Löcher des Nichtwissens" (115) dar. Daher sind Experten, die Sachverstand und Erfahrungswissen bereitstellen, unverzichtbar im Prozess politischer Steuerung. Regieren findet zwar im Spiegel demokratischer Mehrheiten statt, aber es kommt gleichermaßen darauf an, "sich dem Urteil hoch selektiver Fachgemeinschaften und Expertinnen (scientific communities und communities of practice) an[zu]vertauen « (123). Willke sieht hier »eine abgeleitete Form von Legitimität « vorliegen, die er als »Legitimität durch Expertise« (124) bezeichnet.

Abschließend wendet Willke den Blick nach vorn: Die Zukunft des Regierens auf nationalstaatlicher und auf globaler Ebene werde von »einer Verschärfung von Widersprüchen, Ambiguitäten und Ungewissheiten « (135) bestimmt sein. Doch gerade die Vorzüge der Demokratie bestünden darin, »in einem inkrementalen, evolutionären Anpassungsprozess eine zwar kleinteilige, aber kontinuierliche Lernleistung zu erbringen «(136). Diese Aussage sollte man unbedingt als einen hoffnungsvollen Ausblick werten. Trotz der vielfach unvereinbaren Logiken der Teilsysteme verfügen die Einzelteile der Gesellschaft und das politische System über Berührungspunkte, die am Ende auch zu positiven Steuerungsleistungen führen können.

Helge F. Jani 